\title{
A cell-based platform for oxidative stress monitoring in motor neurons using genetically encoded biosensors of $\mathrm{H}_{2} \mathrm{O}_{2}$
}

\author{
Elizaveta I. Ustyantseva ${ }^{1, *}$, Suren M. Zakian ${ }^{1,2,3}$, Sergey P. Medvedev ${ }^{1,2,3,{ }^{*}}$ \\ * Correspondence: e.ustyantseva21gmail.com, medvedev@bionet.nsc.ru
}

1 - The Federal Research Center Institute of Cytology and Genetics, Siberian Branch of Russian Academy of Sciences, 10, Lavrentiev Ave, 630090 Novosibirsk, Russia;

2 - Institute of Chemical Biology and Fundamental Medicine, Siberian Branch of the Russian Academy of Sciences, 8, Lavrentiev Ave., 630090 Novosibirsk, Russia;

3 - E. Meshalkin National medical research center of the Ministry of Health of the Russian Federation, 15 Rechkunovskaya Str., 630055 Novosibirsk, Russia.

\section{ABSTRACT}

Background: Oxidative stress plays an important role in the development of neurodegenerative diseases: it either can be the initiator or part of a pathological cascade leading to the neuron's death. Although a lot of methods are known for oxidative stress study, most of them operate on non-native cellular substrates or interfere with the cell functioning. Genetically encoded (GE) biosensors of oxidative stress demonstrated their general functionality and overall safety in various live systems. However, there is still insufficient data regarding their use for research of disease-related phenotypes in relevant model systems, such as human cells.

Methods: We applied CRISPR/Cas9 genome editing to introduce mutations (c.272A>C and c. $382 \mathrm{G}>\mathrm{C}$ ) in the associated with amyotrophic lateral sclerosis SOD1 gene of induced pluripotent stem cells (iPSC) obtained from a healthy individual. Using CRISPR/Cas9, we modified these mutant iPSC lines, as well as the parental iPSC line, and a patient-specific SODI D91A/D91A iPSC line with ratiometric GE biosensors of cytoplasmic (Cyto-roGFP2-Orp1) and 
mitochondrial (Mito-roGFP2-Orp1) $\mathrm{H}_{2} \mathrm{O}_{2}$. The biosensors sequences along with a specific transactivator for doxycycline-controllable expression were inserted in the "safe harbor" AAVSI (adeno-associated virus site 1) locus. We differentiated these transgenic iPSCs into motor neurons and investigated the functionality of the biosensors in such a system. We measured relative oxidation in the cultured motor neurons and its dependence on culture conditions, age, and genotype, as well as kinetics of $\mathrm{H}_{2} \mathrm{O}_{2}$ elimination in real-time.

Results: We developed a cell-based platform consisting of isogenic iPSC lines with different genotypes associated with amyotrophic lateral sclerosis. The iPSC lines were modified with GE biosensors of cytoplasmic and mitochondrial $\mathrm{H}_{2} \mathrm{O}_{2}$. We provide proof-of-principle data showing that this approach may be suitable for monitoring oxidative stress in cell models of various neurodegenerative diseases as the biosensors reflect the redox state of neurons.

Conclusion: We found that the GE biosensors inserted in the AAVS1 locus remain functional in motor neurons and reflect pathological features of mutant motor neurons, although the readout largely depends on the severity of the mutation.

Keywords: genetically encoded biosensors, oxidative stress, ALS, motor neurons, iPSC, CRISPR/Cas9.

\section{INTRODUCTION}

Redox reactions are part of the cellular metabolism. Normally, reactive oxygen species (ROS), emerging as a byproduct of such reactions, are quickly neutralized by antioxidant systems [1]. In oxidative stress, due to excessive production or disturbed utilization, ROS accumulate, subsequently leading to the cell malfunction [2]. An increasing number of the ROS molecules alter protein structure, change properties of membranes due to lipid peroxidation and cause DNA damage, which allows considering oxidative stress as one of the major mechanisms of degenerative disorders and aging [3,4]. 
It is known that oxidative stress plays an important role in various pathologies, and its involvement in the development of neurodegenerative diseases is indisputable, although not always clear [5-7]. Oxidative stress takes a certain place in amyotrophic lateral sclerosis (ALS) a disorder characterized by the inevitable death of motor neurons resulting in progressive paralysis [8]. The first ALS-associated gene, SOD1, has been discovered in 1993 [9]. SOD1 encodes superoxide dismutase 1, the main component of the antioxidant system; thus, oxidative stress was proposed as the primary pathological mechanism of the disease [9]. Subsequent studies revealed that ALS has a much more complex etiology involving other genes and that only $10 \%$ of the cases are hereditary [10-12]. Moreover, it is considered now that not loss, but gainof-function of mutant SOD1 underlies the ALS development [13]. Nonetheless, signs of oxidative damage have been found in both patients and model organisms, regardless of the initial cause of the disease, suggesting a universal role of redox imbalance in motor neuron damage [14-16].

Redox studies are often conducted by measuring key molecules such as glutathione, hydrogen peroxide, $\mathrm{NADP}^{+} / \mathrm{NADPH}$, and others $[17,18]$. Although dozens of chemical molecular probes have been developed for such analyses, most of them have low specificity, availability and interfere with the cellular processes $[19,20]$. Genetically encoded (GE) biosensors are free from these flaws and can be applied for the same measurement as the molecular probes. GE biosensors are protein-based and delivered inside the cell in the form of nucleic acid. The cell produces biosensor molecules as long as the coding sequence is available. Since the biosensor molecules are produced inside the cell, they do not have the problem of availability, and therefore can be applied not only in cell cultures but also in more complex model systems, such as a whole animal or plant [21-23]. The nature of the genetically encoded biosensors allows easy modifications, e.g. addition of the tags that direct the biosensor to specific cellular compartments (the nucleus, mitochondria, endoplasmic reticulum, and plasma membrane) [24,25]. Traditional methods for the GE biosensors research in cell culture apply their transient expression via 
plasmid delivery or viral-mediated integration of the biosensors sequences [4]. The first approach provides a high-intensity signal but does not allow prolonged experiments. The second one, on the contrary, provides a stable expression of the biosensor but does not guarantee reliable results since randomly integrated biosensors can disturb genome function. Furthermore, some may also speculate that a high level of the biosensor's expression may alter cell functioning due to consumption of the target analytes [19]. Although many redox biosensors have been developed in recent years, only a few have been validated in model systems as suitable for studying disease-associated phenotypes [21,26-28].

Here, we developed a platform for monitoring oxidative stress in motor neurons. We used GE biosensors of cytoplasmic (Cyto-roGFP2-Orp1) and mitochondrial (Mito-roGFP2-Orp1) $\mathrm{H}_{2} \mathrm{O}_{2}$; a known marker molecule that reflects increased ROS production. The biosensors allow ratiometric measurement of hydrogen peroxide, and therefore relative oxidation in the corresponding compartments. The main advantage of the applied approach is that the biosensors' sequences were inserted in the "safe harbor" AAVS1 locus under the control of the doxycyclinedependent promoter, providing prolonged expression of the biosensors and preventing potential negative effect of off-target inserts. To investigate the functionality of the biosensors in these conditions, we generated isogenic induced pluripotent stem cell (iPSC) lines with two mutations affecting different parts of the ALS-associated SOD1 gene. We found that the Cyto-roGFP2Orp1 and Mito-roGFP2-Orp1 biosensors remain functional and reflect $\mathrm{H}_{2} \mathrm{O}_{2}$ levels in iPSCderived motor neurons (Fig 1a). Moreover, we showed that a combination of G128R/K129X mutations, affecting exon 5 of $S O D 1$, results in rapid accumulation of $\mathrm{H}_{2} \mathrm{O}_{2}$ and an aberrant response to exogenous $\mathrm{H}_{2} \mathrm{O}_{2}$ in mature motor neurons, but it was not observed for the D91A mutation.

\section{METHODS}

\section{IPS cell culture}


IPSCs were maintained onto mitotically inactivated mouse embryonic fibroblasts in KnockOut DMEM (Gibco) with 15\% knockout serum replacement (Gibco), $0.1 \mathrm{mM}$ non-essential amino acids (Gibco), penicillin/streptomycin (Lonza), $1 \mathrm{mM}$ GlutaMAX-I, and $10 \mathrm{ng} / \mathrm{mL}$ bFGF at 37 ${ }^{\circ} \mathrm{C}$ and $5 \% \mathrm{CO}_{2}$. IPSC cells were dissociated with TrypLE (Gibco) and split at 1:10 twice a week in the iPSC medium supplemented with $10 \mathrm{ng} / \mathrm{ml} \mathrm{Y-27632.} \mathrm{Original} \mathrm{iPSC} \mathrm{lines} \mathrm{were} \mathrm{derived}$ from the patient (iALS) with a diagnosed hereditary form of ALS [29] and a healthy individual (K7-4Lf) who had no associations with any genetic disease [30] (Supplementary Table 1).

\section{CRISPR/Cas9 design and generation of SOD1 mutant iPSC lines}

The guide RNAs (gRNAs) targeting sequences in exons 4 and 5 of the SODl gene and the AAVS1 locus were designed using the web-based tool http://crispr.mit.edu [31], with a selection of gRNAs with high-quality scores to avoid possible off-targets (Supplementary Table 2). We used CRISPR/Cas9 ribonucleoprotein (RNP) complexes to induce double-strand breaks in the target sites. The Alt-R ${ }^{\circledR}$ crisprRNA and tracrRNA were obtained from IDT (Integrated DNA technologies), and Cas9 protein was expressed in E. coli and purified according to the previously published protocol [32]. The RNP complexes were assembled according to the manufacturer's instructions before the cell transfection. For the introduction of the c.272A $>C$ and c.382G $>C$ mutations we used appropriate SOD1 RNP (20 pmol tracrRNA + 20 pmol crRNA (SOD14/SOD1-5) + 20 pmol Cas9) complexes mixed with 100 pmol of D91A ssODN (single-stranded oligodeoxynucleotide) donor or G128R ssODN donor (Supplementary Table 2). The cells were passed 24 hours before the transfection in the iPSC medium supplemented with Y-27632 (10 $\mathrm{ng} / \mathrm{ml})$. On the day of transfection, the cells were dissociated with TrypLE, strained through a 70 $\mu \mathrm{m}$ cell strainer (Miltenyi biotec), counted, centrifuged at $200 \mathrm{~g}$ for $5 \mathrm{~min}$, and resuspended in $\mathrm{R}$ buffer (Neon Transfection System, Invitrogen) according to the manufacturer's instructions. 10 ul of the suspension was taken to the electroporation by Neon Transfection System with the following impulse settings: $1100 \mathrm{~V}, 30 \mathrm{~ms}, 1$ pulse. The cells were seeded onto feeder-coated 4 
$\mathrm{cm}^{2}$ dishes in the iPSC medium supplemented with Y-27632 $(10 \mathrm{ng} / \mathrm{ml})$. The next day, the cells were dissociated with TrypLE, strained through the cell strainer, and subcloned on three 96-well plates three cells per well for propagation and analysis. Genomic DNA of the survived clones was obtained and analyzed for the presence of the target mutations.

\section{Screening of iPSC clones for the c.272A >C (D91A) and c.382G >C (G128R) substitutions}

To detect the c.272A $>\mathrm{C}$ mutation, we designed primers for tetra-primer ARMS (amplificationrefractory mutation system) PCR screening using http://primer1.soton.ac.uk/primer1.html (Supplementary Table 2) [33] and performed touchdown 3-step PCR: annealing at $68-64{ }^{\circ} \mathrm{C}$ for 9 cycles, then at $64{ }^{\circ} \mathrm{C}$ for 21 cycles. The PCR products were analyzed in $2 \%$ agarose gel. Clones positive for mutant allele presence were further examined by Sanger sequencing (Supplementary Table 2). To detect the c.382G $>$ C mutation, we designed a pair of primers that amplify the target locus of the SOD1 gene and two fluorescent probes targeting either wild-type or mutant sequence (Supplementary Table 2). Using LightCycler 480 (Roche), we analyzed the clones and selected those who had strong signals from the mutant-targeted probe. The target mutation was further confirmed by Sanger sequencing (Supplementary Table 2). The clones used in the experiments were characterized according to the Human Pluripotent Stem Cell Registry standards with the protocols described earlier [34].

\section{Generation, selection, and screening of iPSC clones with target $A A V S 1$ inserts}

To insert Cyto-roGFP2-Orp1, Mito-roGFP2-Orp1 and transactivator in AAVS1, we used AAVS1 RNP (100 pmol tracrRNA + 100 pmol AAVS1 crRNA + 100 pmol Cas9) mixed with $5 \mu \mathrm{g}$ of donor plasmids mix, containing equimolar amounts of transactivator donor (pAAVS1-NeoM2rtTA, Addgene \# 60843) + pCyto-roGFP2-Orp1-donor or pMito-roGFP2-Orp1-donor. The cells were prepared as it was described earlier and resuspended in R buffer. We mixed $100 \mu 1$ of the cell suspension, RNP complexes and donor plasmids and performed transfection using Neon 
Transfection System $(2 \times$ reaction per experiment). The cells were then seeded onto feeder-coated $10 \mathrm{~cm}^{2}$ dishes in the iPSC medium supplemented with $\mathrm{Y}-27632(10 \mathrm{ng} / \mathrm{ml})$ and maintained until small colonies formed, for 2-3 days prior to the selection. For the selection of subclones with the target biosensor and transactivator inserts, we supplemented the iPSC medium with puromycin dihydrochloride (Sigma-Aldrich) for 3 days. Then we replaced the antibiotic with neomycin sulfate (Sigma-Aldrich) and incubated the cells for 4-5 more days. Antibiotics concentrations were determined for each cell line by titration before the experiment. At the end of the selection, we added doxycycline hyclate $(2 \mu \mathrm{g} / \mathrm{ml}$, Sigma-Aldrich) and examined the remained clones for the presence of fluorescent signal from the biosensors' roGFP2 (reduction-oxidation sensitive green fluorescent protein 2) using the Nikon Eclipse Ti2-E (Nikon) microscope. The clones positive for the roGFP2 expression that survived double antibiotic selection were manually harvested into separate dishes for maintaining and analysis. We extracted genomic DNA from these iPSC clones and analyzed for the presence of the target and off-target inserts of the donor plasmids using PCR with specific primers (Supplementary Table 2).

\section{Immunocytochemistry}

Immunocytochemistry was performed on iPSCs and motor neurons (ChAT - differentiation day 20 and 28; ISL1 and MNX1 - differentiation day 28). The cells were fixed in 4\% formaldehyde solution (Sigma-Aldrich) for $10 \mathrm{~min}$ at room temperature (RT), permeabilized with $0.5 \%$ Triton X-100 (Sigma-Aldrich) for $30 \mathrm{~min}$ at RT, and then incubated with blocking buffer (1\% bovine serum albumin (BSA) in PBS, Sigma) for $30 \mathrm{~min}$ at RT. After, the cells were incubated with specific primary antibodies overnight at $4{ }^{\circ} \mathrm{C}$. The appropriate secondary antibodies were added for 1.5-2 $\mathrm{h}$ incubation at RT. All antibodies were diluted in blocking buffer, and the cell nuclei were visualized with DAPI ( $1 \mu \mathrm{g} / \mathrm{ml}$ solution in PBS; Sigma-Aldrich). The antibodies and their dilution ratios are listed in the Supplementary Table 3. Micrographs were captured using either 
Nikon eclipse Ti-E microscope (Nikon) and NIS Elements software or LSM-780 (Zeiss) microscope and ZEN black software.

\section{Motor neuron differentiation}

The iPSCs were seeded onto dishes coated with Matrigel-ESQ (Corning) in E8 (Gibco) medium and maintained in feeder-free conditions for at least 2 passages prior to differentiation. Motor neuron differentiation was performed according to the previously published 4-step protocol [35]. For neural patterning, the E8 medium was changed to basal neuronal differentiation medium (NDM): F12/DMEM:Neurobasal - 50:50, 0.5× N2 supplement, 0.5× B-27 supplement, $2 \mathrm{mM}$ GlutaMAX, and $0.1 \mathrm{mM}$ ascorbic acid. At the first step of differentiation, the NDM was supplemented with $2 \mu \mathrm{M}$ CHIR99021 (StemRD), $2 \mu \mathrm{M}$ SB431242 (Selleckchem), and $2 \mu \mathrm{M}$ DMH1 (Tocris) for 6 days. Then the cells were dissociated with Accutase (Gibco) and plated 1:6 onto Matrigel-coated surface in the NDM medium, supplemented with CHIR99021, SB431242, DMH1, $0.5 \mu \mathrm{M}$ Purmorphamine (Stegment), and $0.1 \mu \mathrm{M}$ retinoic acid (Sigma) for another 6 days (the second step). After, the cells were dissociated with Accutase and cultured in the suspension on agarose-coated (non-adherent) dishes in the NDM medium, supplemented with $0.1 \mu \mathrm{M}$ Puromorphamin and $0.5 \mu \mathrm{M}$ retinoic acid for 6 days (the third step). Then the cells were dissociated with Accutase to a single-cell suspension, strained through the $70 \mu \mathrm{m}$ cell strainer, and seeded in the NDM medium, supplemented with $0.1 \mu \mathrm{M}$ Puromorphamin, $0.5 \mu \mathrm{M}$ retinoic acid, and 0.15 $\mu \mathrm{M}$ Compound E (EMDMillipore) either on Matrigel-coated surfaces or inside a layer of the Matrigel for 10-day maturation (fourth step). Depending on the experiment, the cells were seeded in different settings. For flow cytometry and mRNA expression analysis, we seeded $1.5 \times 10^{5}$ cells $/ \mathrm{cm}^{2}$ onto Matrigel-coated $60 \mathrm{~mm}$ Petri dishes; for immunocytochemistry $-5 \times 10^{4}$ cells $/ \mathrm{cm}^{2}$ - onto Matrigel-coated cell imaging coverglasses (Eppendorf); for axon measurement $1.5 \times 10^{4}$ cells $/ \mathrm{cm}^{2}-$ onto Matrigel-coated cell imaging coverglasses. After dissociation between each step, cells were transferred in the NDM medium, supplemented with Y-27632 (10 ng/ml). 
The medium was replaced every day. The cells were supplemented with doxycycline $(2 \mu \mathrm{g} / \mathrm{ml})$ every other day during the differentiation unless otherwise indicated.

For starvation induction, we used Neuronal Deficit Medium (NDefM, F12/DMEM:Neurobasal 50:50, 1x N2 supplement, $2 \mu \mathrm{g} / \mathrm{ml}$ doxycycline hyclate). For antioxidant-deprivation assay and excitotoxicity induction assay, $\mathrm{MN}$ were incubated in the neuronal maintenance medium without antioxidants (F/D:Neurobasal $-50: 50,0.5 \times \mathrm{N} 2$ supplement, 0.5× B-27 Supplement without antioxidants), supplemented with $0.5 \mu \mathrm{M}$ retinoic acid, and $0.15 \mu \mathrm{M}$ Compound $\mathrm{E}$.

\section{Preparation of live motor neuron samples for microscopy}

Motor neurons have a low surface attachment, which makes prolonged microscopy experiments difficult. Therefore, in the biosensors experiments, the cells were seeded for maturation on the cell imaging coverglasses inside a layer of 33\% Matrigel. To do so, we resuspended the cells in the $1.5 \times$ cold fourth step medium (supplemented with $15 \mathrm{ng} / \mathrm{ml} \mathrm{Y-27632),} \mathrm{making} \mathrm{a} \mathrm{suspension}$ with $1.5 \times 10^{5}$ cells $/ 75 \mu \mathrm{l}\left(1.5 \times 10^{5}\right.$ cells/well). Then, we mixed $75 \mu 1$ of the cell suspension with $35 \mu \mathrm{l}$ of growth factor reduced Matrigel (Corning) and quickly applied $100 \mu \mathrm{l}$ of the mix onto the surface of the chilled cell imaging coverglass, standing on a cold tube cooling rack turned upside down and covered with a paper towel. Using the tip of a pipette, we carefully spread the mix over the surface and left it on the cooling rack for 10 minutes to let the cells fall to bottom before the Matrigel polymerized. After, we transferred the coverglasses on top of the working surface and left them for another 10 minutes, and, then, the coverglasses were carried over to a $\mathrm{CO}_{2}$ incubator for $1 \mathrm{~h}$ for Matrigel stabilization. After 1 hour, we added 300-400 $\mu \mathrm{l}$ of the fourth step medium supplemented with Y-27632 $(10 \mathrm{ng} / \mathrm{ml})$ on top of the stabilized Matrigel layer (Supplementary Fig. S1). 


\section{Reverse-transcription quantitative PCR (RT-qPCR)}

Total RNA was extracted from iPSC and motor neurons with Trizol reagent (Invitrogen). The reverse transcription of $1 \mu \mathrm{g}$ of total RNA was performed with 5x RT-buffer mix with M-MuLVRH reverse transcriptase (Biolabmix) and random hexamer primer (Invitrogen) and diluted 1:10 in MilliQ $\mathrm{H}_{2} \mathrm{O}$. To measure the endogenous gene expression of pluripotency factors, motor neurons' markers, and transgene expression the qPCR analysis was performed using LightCycler 480 (Roche). Gene expression of the iPSC markers in the SOD1 mutant iPSCs was normalized to the $B 2 M$ housekeeping gene and compared to the original iPSC line using the $\Delta \Delta \mathrm{Ct}$-method. ISL1, CHAT, and MNX1 genes expression in motor neurons was normalized to the mean of the GAPDH, HPRT1, and RPL13 housekeeping genes and compared to expression in iPSC sample using the $\Delta \Delta \mathrm{Ct}$-method. The roGFP2 and rtTA transgenes expression in motor neurons and iPSCs was normalized to the mean of GAPDH, HPRT1, and RPL13 housekeeping genes and compared to the expression in motor neuron sample that was not treated with doxycycline during differentiation. The primers used are listed in the Supplementary Table 2.

\section{Flow cytometry analysis}

To identify the proportion of motor neurons in the differentiation, we dissociated the cells with Accutase on the day 20 of the differentiation protocol, resuspended in cold PBS, and centrifuged at $400 \mathrm{~g}$ for 5 minutes (the same settings were used for all subsequent centrifugation steps). The pellet was resuspended in $1 \mathrm{ml}$ cold $4 \%$ formaldehyde solution and incubated on ice for 10-15 minutes. After, we added $1 \mathrm{ml}$ cold PBS, centrifuged the cells, discarded supernatant, resuspended the pellet in $1 \mathrm{ml}$ ice-cold $100 \%$ methanol, and incubated it for 10-15 minutes on ice. Then, the pellet was washed twice with flow cytometry staining buffer $(1 \% \mathrm{BSA}, 0.2 \mu \mathrm{M}$ EDTA, in PBS) and resuspended in it to $1 \times 10^{6}$ cells $/ \mathrm{ml}$ concentration. $100 \mu \mathrm{l}$ of the cell suspension was incubated with anti-ISL primary antibodies overnight at $4{ }^{\circ} \mathrm{C}$. The cells were washed with the flow cytometry staining buffer and incubated with the secondary antibodies for 
30 minutes at RT. Cells were analyzed using FACSAria (BD Biosciences). Unlabeled cells and isotype-labeled cells were used as controls.

\section{Fluorescence intensity measurement}

To measure the biosensors' fluorescence intensity, we obtained images of the MN using a Zeiss LSM-780 confocal laser scanning microscope (Pan-Apochromat 20× objective) adjusted for visualization of a green dye: excitation with $488 \mathrm{~nm}$ argon laser, emission collection at 500-530 nm. Using the ImageJ software, we measured the mean intensity of fluorescence on each image and calculated corrected total cell fluorescence $(\mathrm{CTCF})$ with the formula: $\mathrm{CTCF}=$ Integrated Density - (Area occupied by cells $\times$ Mean fluorescence of background readings) to reduce the background contribution. The results were obtained from six separate experiments, which are represented by the MN derived from different iPSC clones.

\section{Axon measurement}

The immature motor neurons were seeded on the cell imaging coverglasses in a low density $\left(1.5 \times 10^{4}\right.$ cells $\left./ \mathrm{cm}^{2}\right)$ and grown for 2 days in the fourth step medium supplemented with neurotrophic factor (NTF) cocktail: IGF1 (PeproTech, 10 ng/ml), CNTF (PeproTech, 10 ng/ml), BDNF (PeproTech, $10 \mathrm{ng} / \mathrm{ml}$ ). Doxycycline $(2 \mu \mathrm{g} / \mathrm{ml}$ ) was added to induce the roGFP2 expression. RoGFP2 served as a label, marking the cellular contour and processes of the live neurons. We obtained mages for each cell line with the Nikon Eclipse Ti-2E microscope (20× objective, FITC channel). Using ImageJ, we manually measured the length of the longest processes (axons) of free-lying neurons with visible ends. Only axons with the length more than twice the size of the neurons' bodies were considered for the analysis. If the neuron had two long processes, the longest one was considered for the measurement. The mean length of the axons was calculated based on the data obtained from the differentiation of three separate iPSC clones for each genotype present. 


\section{Image acquisition}

The general procedure for the redox biosensors measurement was described earlier by B. Morgan and colleagues [36]. Although, we modified the protocol to be more suitable for cultured motor neurons.

\section{Cells and solutions preparation}

To measure $\mathrm{H}_{2} \mathrm{O}_{2}$ utilization in real-time, we replaced the medium in the neurons with the neuronal deficit medium the day before the experiment, unless otherwise indicated. 1 hour before the experiment the medium has been removed from the analyzed wells (so as not to disturb the Matrigel layer with neurons inside), and replaced with warm $\mathrm{HBSS}+\mathrm{Ca}^{2+}, \mathrm{Mg}^{2+}$. The cells were incubated in $\mathrm{CO}_{2}$ incubator for removal of the residual medium components from the Matrigel layer. Then, the old HBSS has been almost completely removed (with residual of $\sim 50 \mu 1 /$ well) and replaced with the fresh HBSS $(\sim 300 \mu \mathrm{l} /$ well $)$.

The stock and working solutions were prepared freshly on the day of the experiment. The water stock solutions of $1 \mathrm{M}$ DTT (Sigma-Aldrich) and 0.2 M diamide (Sigma-Aldrich) were made from a powder; $10 \mu \mathrm{M} \mathrm{H}_{2} \mathrm{O}_{2}$ stock solution was made from hydrogen peroxide solution (30\% w/w, Sigma-Aldrich).

From these stock solutions, the working solutions has been prepared:

1) $10 \mu 111 \mathrm{M} \mathrm{DTT}+190 \mu \mathrm{l} \mathrm{HBSS}$;

2) $5 \mu \mathrm{l} 0.2 \mathrm{M}$ diamide $+195 \mu \mathrm{l} \mathrm{HBSS}$;

3) $2 \mu \mathrm{l} 10 \mu \mathrm{M} \mathrm{H}_{2} \mathrm{O}_{2}+198 \mu \mathrm{lHBSS}$.

\section{Microscopy settings}

We used a confocal Zeiss LSM-780 laser scanning microscope (Pan-Apochromat 20× objective) equipped with the $488 \mathrm{~nm}$ argon laser, the $405 \mathrm{~nm}$ UV diode laser, and a climate chamber connected with the temperature and $\mathrm{CO}_{2}$ control modules. Cell imaging coverglass with the cells was placed on the stage without a lid, covered with a $\mathrm{CO}_{2}$ cover, and left for 5-10 minutes for temperature equilibration. Tubes with the working solutions were also put inside the climate 
chamber. During the equilibration, the cells were visualized with transmitted light to achieve a stable focus. Actual microscopy settings varied between the samples and required customization for obtaining a quality image. Table 1 describes the initial microscopy setup. An image did not contain oversaturated pixels and had a high noise-to-signal ratio.

Table 1. Initial microscopy setup (Zeiss LSM-780) for imaging of Cyto-roGFP2-Orp1 and MitoroGFP2-Orp1 expressing motor neurons

\begin{tabular}{|c|c|}
\hline Mode & Channel mode \\
\hline \multicolumn{2}{|l|}{ Channels: } \\
\hline Switch track every & «Frame» \\
\hline Track 1 & $\begin{array}{l}405 \mathrm{~nm} \text { (DAPI, roGFP2ox) }+ \text { T-PMT } \\
\text { (Transmission) }\end{array}$ \\
\hline Track 2 & $488 \mathrm{~nm}$ (EGFP, roGFP2red) \\
\hline \multicolumn{2}{|l|}{ Light Path: } \\
\hline Track $1+$ Track 2 & $500-530 \mathrm{~nm}$ \\
\hline \multicolumn{2}{|l|}{ Acquisition mode: } \\
\hline Scan mode & Frame \\
\hline Frame size & $512 \times 512(1024 \times 1024$ for photo $)$ \\
\hline Scanning speed (pixel dwell) & $9(1,58 \mu \mathrm{sec})$ \\
\hline Averaging & 1 \\
\hline Bit depth & 16 bit \\
\hline Zoom & 0.6 \\
\hline \multicolumn{2}{|l|}{ Channel/Laser settings: } \\
\hline 405-nm line & \\
\hline Attenuator (transmission) & $5 \%$ \\
\hline Pinhole & 74.5 \\
\hline
\end{tabular}




\begin{tabular}{|c|l|}
\hline Gain & 800 \\
\hline Digital gain & 1 \\
\hline 488 -nm line & \\
\hline Attenuator (transmission) & $2 \%$ \\
\hline Pinhole & 74.5 \\
\hline Gain & $800-$ Cyto-roGFP2-Orp1, 700 - Mito-roGFP2- \\
& Orp1 \\
\hline Digital gain & 1 \\
\hline
\end{tabular}

\section{Biosensor calibration using DTT/diamide}

To determine the states of the maximum oxidation and reduction possible for the biosensors, we treated the cells with diamide and DTT, respectively, using the following procedure:

1) In the mode "Position" set up three fields of view in each of the two adjacent wells and saved their coordinates;

2) Set the microscope to the "time series" mode with 18 cycles of $90 \mathrm{~s}$;

3) Started the time series experiment and paused it after cycle 3; added $50 \mu 1$ of the warm DTT working solution into one well (final concentration $5 \mu \mathrm{M}$ ) and $50 \mu$ of the warm diamide working solution into the other well (final concentration $0.5 \mu \mathrm{M}$ ), trying not to disturb the coverglass and the cell layer; resumed the time series;

4) Waited for the cells to become fully reduced/oxidized (usually 8-10 minutes): the signal in each sample reached the plateau. In the DTT well intensity of the $488 \mathrm{~nm}$ signal slightly increased, while intensity of the $405 \mathrm{~nm}$ signal slightly decreased; in the diamide well intensity of the $488 \mathrm{~nm}$ signal intensity considerably decreased, while intensity of the $405 \mathrm{~nm}$ signal considerably increased. 
The calibration procedure has been performed for every sample before the other experiments, and the values of the maximum oxidation/reduction were used for the dynamic range calculation and data normalization.

The basal $\mathrm{H}_{2} \mathrm{O}_{2}$ level measurement and the measurement of $\mathrm{H}_{2} \mathrm{O}_{2}$ utilization in real-time.

For the measurement of the Cyto-roGFP2-Orp1 and Mito-roGFP2-Orp1 signals in motor neurons, we applied the microscope settings determined during the calibration. For the measurement of basal $\mathrm{H}_{2} \mathrm{O}_{2}$ levels in cytoplasm in mitochondria, we obtained images from $\mathrm{MN}$ derived from the K7-4Lf, SOD1-D91A, SOD1-G128R, and iALS iPSC lines. Starvation was induced by changing the medium to the neuronal deficit medium. Rescue of the SOD1-G128R MN was performed by the addition of a neurotrophic factor (NTF) cocktail: IGF1 (10 ng/ml), CNTF $(10 \mathrm{ng} / \mathrm{ml})$, and BDNF (10 ng/ml) to the culture medium. To measure $\mathrm{H}_{2} \mathrm{O}_{2}$ utilization in real-time, we applied the same approach as for calibration, but with 33 cycles, each 3 minutes long. We prepared the cells as described, mounted the coverglasses on the stage, and equilibrated the temperature in the climate chamber. We added $50 \mu \mathrm{l}$ of the warm working $\mathrm{H}_{2} \mathrm{O}_{2}$ solution (final concentration $10 \mu \mathrm{M}$ ) to the cells after cycle 2-3, and then continued the time series for another 30 cycles (1.5 hours).

\section{Excitotoxicity induction assay}

To induce glutamate-mediated excitotoxicity, we incubated $\mathrm{MN}$ in the neuronal maintenance medium supplemented with $0.5 \mu \mathrm{M}$ Retinoic acid, $0.15 \mu \mathrm{M}$ Compound E, $20 \mu \mathrm{M}$ monosodium glutamate (Sigma-Aldrich), and $100 \mu \mathrm{M}$ 1-trans-pyrrolidine-2,4-dicarboxylic acid (PDC, SigmaAldrich) for 5 days, changing the medium every other day. After 5 days of incubation, we obtained images of the treated $\mathrm{MN}$ and non-treated control MN. The data obtained at the end of the experiment were normalized to the starting oxidation values, measured before the glutamate addition, to describe the changes that emerged during the experiment. 


\section{Data normalization}

The images were saved as 16-bit .tiff files and processed by ImageJ. For the analysis, the images were converted to the 32-bit format. Single pictures were split to the $405 \mathrm{~nm}$ (roGFP $\left.2_{\text {ox }}\right), 488 \mathrm{~nm}$ (roGFP $2_{\text {red }}$, and transmitted light channels. The intensity of the 405 and 488 channels was thresholded, and values below the threshold were set to "not a number" $(\mathrm{NaN})$. A ratio image was created by dividing the $405 \mathrm{~nm}$ image to the $488 \mathrm{~nm}$ image (roGFP $2_{\text {ox }} / \mathrm{roGFP} 2_{\text {red }}$ ), and the mean intensity of the resulting image was measured. The time series images were processed similarly. The files were imported in ImageJ as stacks, converted to the 32-bit format, split using the "Stacks-Shuffling-Deinterleave" plugin. The threshold was adjusted for both channels, and the values below the threshold were set to "not a number" $(\mathrm{NaN})$. A resulting ratio images were created by dividing the $405 \mathrm{~nm}$ image to the $488 \mathrm{~nm}$ and measured. The roGFP $2_{\mathrm{ox}} / \mathrm{roGFP} 2_{\text {red }}$ ratios of the single images were used to describe the basal level of $\mathrm{H}_{2} \mathrm{O}_{2}$. The roGFP $2_{\text {ox }} /$ roGFP $2_{\text {red }}$ ratios obtained from the time series were used for measurement of $\mathrm{H}_{2} \mathrm{O}_{2}$ utilization in real-time. Data obtained from different lines were normalized to a fraction of the maximum oxidation/reduction state according to the formula (1):

Normalized $\frac{\text { roGFP2ox }}{\text { roGFP2red }}=$ Sample $\frac{\text { roGFP2ox }}{\text { roGFP2red }}-$ DTT $\frac{\text { roGFP2ox }}{\text { roGFP2red }} /$ Diamide $\frac{\text { roGFP2ox }}{\text { roGFP2red }}-$ DTT $\frac{\text { roGFP2ox }}{\text { roGFP2red }}$

The normalized data were used for visualization, comparison and statistical analysis.

\section{Maximum oxidation and recovery rate calculation}

The maximum biosensor's oxidation was calculated by subtraction of the initial normalized value of the roGFP2ox/roGFP2red ratio recorded at " 0 " time point from the normalized maximum oxidation value of the roGFP2ox/roGFP2red ratio obtained after $\mathrm{H}_{2} \mathrm{O}_{2}$ addition (usually after 30 minutes of recording). The recovery rate was calculated by subtraction of the final normalized value of the roGFP2ox/roGFP2red ratio recorded at the end of the time series 
from the normalized maximum oxidation value with subsequent division of the resulting numbers to the time interval between these two time points (in hours).

\section{Statistics}

The data derived from at least three different clones of the same genotype were collected for the comparisons; except for measurements of $\mathrm{H}_{2} \mathrm{O}_{2}$ utilization in starvation assay and Mito-roGFP2Orp1 reaction to $\mathrm{H}_{2} \mathrm{O}_{2}$, where data were collected from the $\mathrm{MN}$ derived from one iPSC clone. Analysis of the axon length was performed using one-way ANOVA with the Bonferroni correction for multiple comparisons. Analyses of mRNA expression, fluorescence intensity and all the information obtained from the biosensors were performed using the Mann-Whitney U-test with the Bonferroni correction for multiple comparisons where necessary. Averaged data are represented in the form of scatter plots, or line graphs as the mean \pm standard error of the mean (the mean \pm S.E.M) or as the mean \pm standard deviation (the mean \pm S.D.). The number of experimental replicates is denoted as $\mathrm{N}$. Statistical significance is defined as $* \mathrm{p}<0.05, * * \mathrm{p}<$ $0.01, * * *$ (or \#\#\#) $\mathrm{p}<0.001, * * * *$ (or \#\#\#\#) p $<0.0001$.

\section{RESULTS}

\section{Introduction of the SOD1 D91A and G128R mutations in IPSCs of the clinically healthy}

\section{donor}

It is known that SOD1 has more than 140 mutations associated with ALS, and they define clinical features of the disease such as its manifestation age, rate of symptoms progression, presence of additional neurological symptoms, etc [37]. Several theoretical studies [38,39] hypothesized that severity of the symptoms is highly dependent on the position of a mutation in the sequence and its effect on the protein folding and stability. We designed two CRISPR/Cas9 guide RNA targeting the sequences in the exons 4 and 5 of SOD1 and two ssODN donor templates necessary for the introduction of c.272A $>C$ and c.382G $>C$ single nucleotide mutations 
that lead to the D91A and G128R substitutions, respectively, in the SOD1 polypeptide (Fig. 1b, Supplementary Table 2). The c.272A>C mutation is known for its relatively mild character with late manifestation and long progression, while c.382G $>\mathrm{C}$ is characterized by extremely rapid development $[40,41]$.

We introduced these mutations using CRISPR/Cas9, into a well-characterized control iPSC line (K7-4Lf), obtained earlier from a clinically healthy individual [30] (Supplementary Table 1) and recovered 66 clones for D91A variant and 124 clones for G128R variant. We screened those clones by tetra-primer ARMS PCR (for D91A) or qPCR with fluorescent probes (for G128R) and found $6(9.1 \%)$ and $4(3.2 \%)$ clones, respectively, presumably positive for the target mutations. The target mutations were further confirmed by Sanger sequencing. As a result, a number of clones with different SOD1 allelic variants were obtained (Fig. 1c). Since no homozygous clones were found, we chose clones with SOD1 $1^{\text {91A/del105 }}$ (SOD1-D91A) and SOD1 $1^{G 128 R / K 129 *}$ (SOD1-G128R) variants for subsequent experiments. It was suggested that the destruction of one of the alleles will create a more severe phenotype and make biosensors' measurements more robust. These iPSC lines demonstrated features specific for pluripotent cells: they expressed specific genes and proteins (OCT4, NANOG, SOX2, and TRA1-60), positively stained for alkaline phosphatase, and were able to generate three germ layer derivatives. The cells also retained a normal 46, XX karyotype and were free of mycoplasma contamination (Fig.

\section{1d-e, Supplementary Fig. S2).}


bioRxiv preprint doi: https://doi org/10.1101/2021.0913459724; this version posted September 15,2021 . The copyright holder for this preprint (which was not certified by peer review) is the author/funder, who has granted bioRxiv a license to display the preprint in perpetuity. It is made available under aCC-BY 4.0 International license.

A

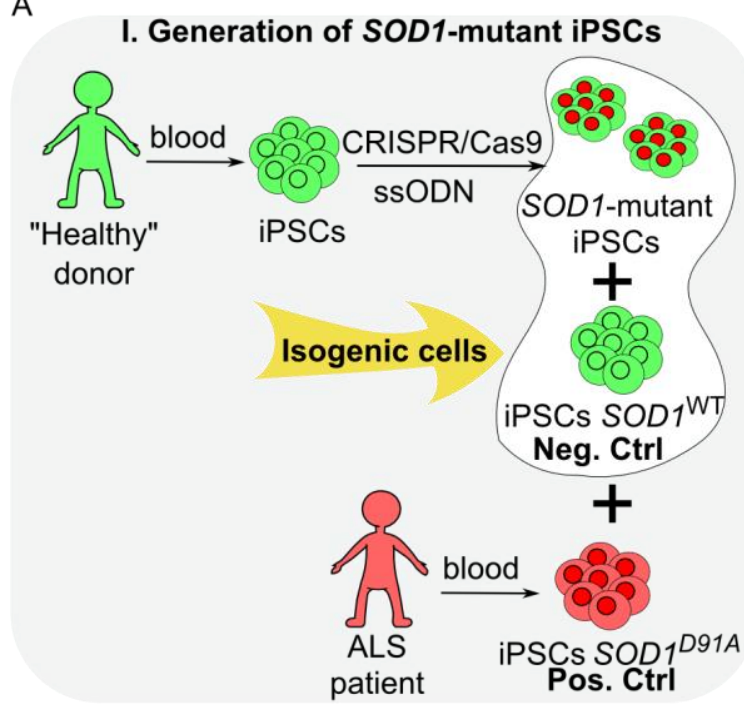

B

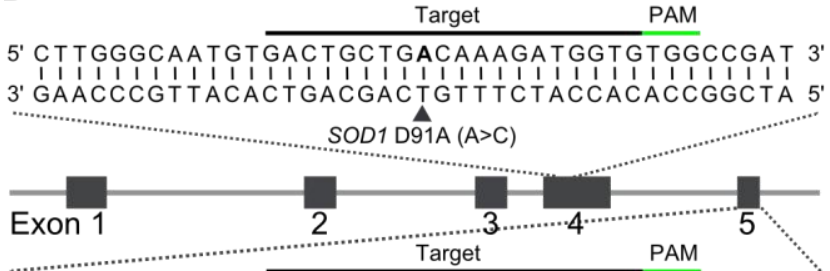

5' GTTCATGAAAAAGCAGATGACTTGGGCAAAGGTGGAATG 3 3. CAIIIIIIIIIIIIIIIIIIIIIIIIIIIIIIIIIIIIIIII SOD1 G128R (G>C)

D

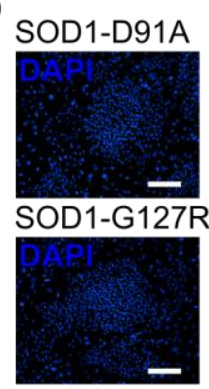

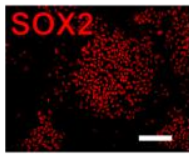

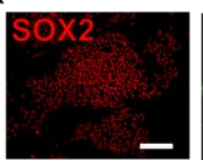

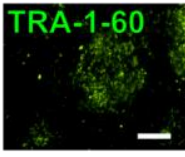

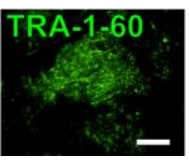

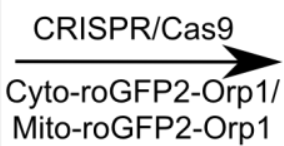

donor
II. Generation of biosensors' expressing motor neurons

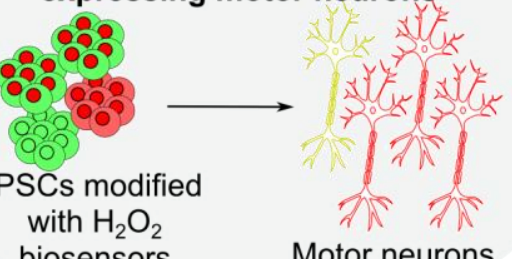

biosensors

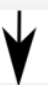

III. Characterisation and analysis

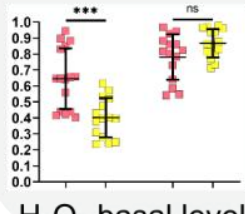

$\mathrm{H}_{2} \mathrm{O}_{2}$ basal leve

C

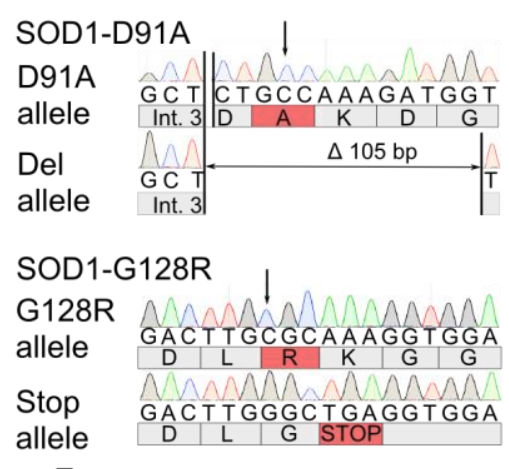

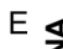

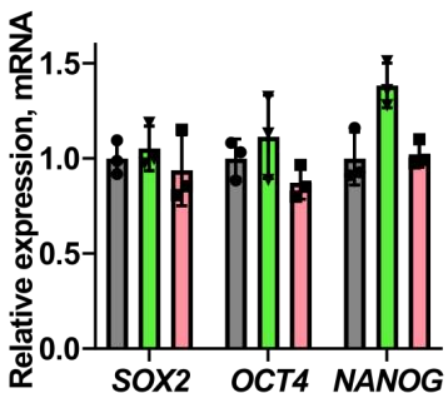

Alternative variants: SOD1 D91AWT SOD $1^{D 91 A / \triangle 5 b . p .}$ SOD1 $1^{\text {D91A/ins } 1 \text { b.p. }}$ SOD $1^{G 128 R / \Delta 5 \text { b.p. }}$ SOD $1^{\text {G128R/ }}$ 9b.p. SOD1 1 128R/ins.1b.p.

K7-4Lf SOD1-D91A $\square$ SOD1-G128R

Fig. 1. Generation of isogenic SODl-mutant iPSC lines. A Schematic representation of the experimental design. iPSCs - induced pluripotent stem cells; Neg. Ctrl - negative control; Pos. Ctrl - positive control; ssODN - singlestranded oligodeoxynucleotide. B Schematic of the SOD1 gene with partial sequences of the exons 4 and 5 . Protospacers designed for CRISPR/Cas9-mediated double-stranded breaks are underlined with black lines, PAM with green lines; target mutations are in bold and marked with black triangles. C Partial SOD1 sequences of the exons 4 and 5 of the SOD1-D91A and SOD1-G128R iPSC lines. Int. 3 - intron 3. In the box: list of clones with an alternative SOD1 variants obtained in the study. D Immunocytochemistry of the SOD1-D91A and SOD1-G128R iPSC lines. The cells are positively stained for pluripotency markers: transcriptional factor SOX2 and surface antigen TRA-1-60. Nuclei are visualized with DAPI, scale bar $100 \mu \mathrm{m}$. E RT-qPCR analysis of mRNA expression of SOX2, OCT4, and NANOG in the SOD1-D91A and SOD1-G128R iPSCs. Data $(\mathrm{N}=3)$ are normalized to the 
parental iPSC line (K7-4Lf) and presented as the mean \pm standard deviation. Characterization of the SOD1-D91A and SOD1-G128R iPSC lines in detail is in the Supplementary Fig. S2.

\section{Generation of transgenic IPSC lines with the inserts of genetically encoded biosensors of $\mathrm{H}_{2} \mathrm{O}_{2}$ via CRISPR/Cas9-mediated $A A V S 1$ targeting}

We introduced the sequences of two genetically encoded biosensors: Cyto-roGFP2-Orp1 and Mito-roGFP2-Orp1, measuring $\mathrm{H}_{2} \mathrm{O}_{2}$ levels in the cytoplasm and mitochondria, respectively, in the genome of SOD1-D91A, SOD1-G128R, and the isogenic control line (K7-4Lf) to obtain stable expression. Additionally, we introduced the same sequences in the genome of a patientspecific iPSC line (iALS) previously generated in our lab from the patient, diagnosed with a hereditary form of ALS with homozygous D91A mutation in SOD1 [34]. This cell line does not share the same genetic background with the SOD1-mutant and control iPSC lines but serves as an external positive control.

The Tet-On expression system applied for the biosensors' expression consists of two elements [42]: the biosensor's sequence that follows the tetracycline-dependent promoter and the specific transactivator (rtTA, reverse tetracycline-controlled transactivator) essential for the controlled expression of the target genes. To deliver these elements in the cell's genome, we used biallelic target insertion in the safe harbor AAVS1 locus (the first intron of the PPP1R12C gene) via CRISPR/Cas9 (Fig. 2a). The donor plasmid containing the rtTA, homologous arms, and neomycin resistant gene for the selection was obtained from the vendor (Addgene) [43]. The biosensors' donor plasmids were designed and constructed in the lab [29]. These donor plasmids contained the tetracycline-dependent promoter, followed by the Cyto-roGFP2-Orp1 and MitoroGFP2-Orp1 sequences, as well as $~ 800$ base long homology arms, and puromycin resistance gene for the selection of the target clones (Supplementary Fig. S3). We delivered both donor plasmids along with the CRISPR/Cas9 RNPs in the IPSCs and selected them using appropriate media supplemented with neomycin and puromycin. Among the survived clones, we manually picked up and subcloned those who positively responded to the doxycycline (tetracycline 
derivative) addition with the biosensors expression and screened the clones for the presence of target biallelic insertion in the AAVS1 locus by PCR (Fig. 2b-c). Further, clones positive for the target insertions were screened for additional off-target donor integration by PCR using specific pairs of primers that only detect non-integrating parts of the donor plasmids, implying that the presence of the PCR-product indicates an off-target insertion. We obtained from 3 to 17 separate iPSC clones positive for the target and negative for the off-target insertions for each cell line (Fig. 2d). All transgenic iPSC clones used further in the experiments were stained positive for the specific pluripotent cell markers SOX2 and SSEA4 (Supplementary Fig. 4).

A

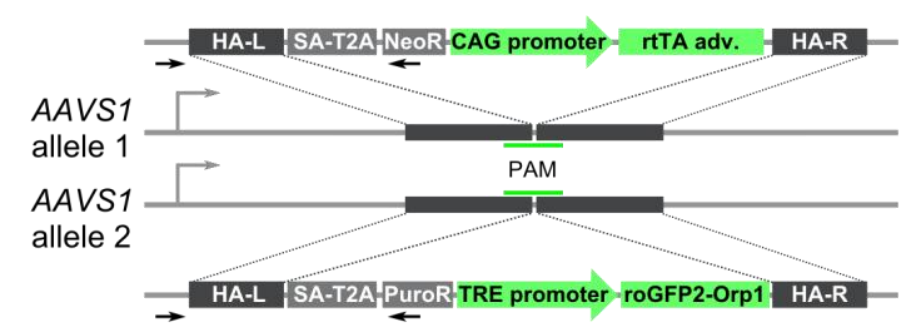

C

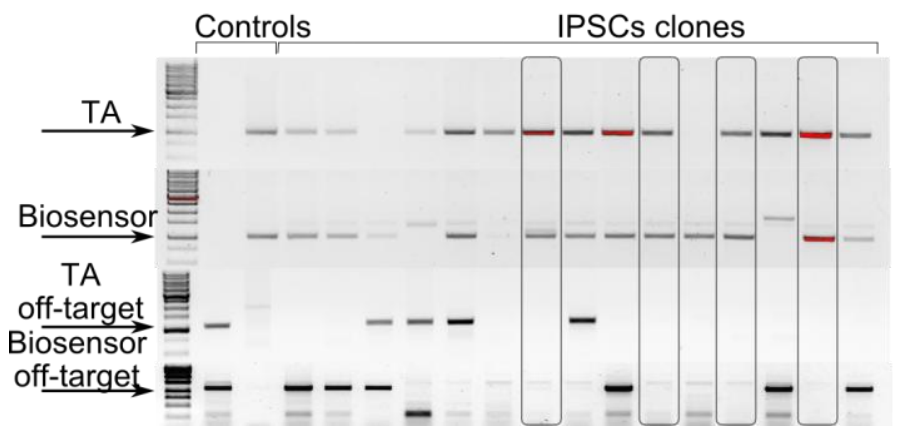

B

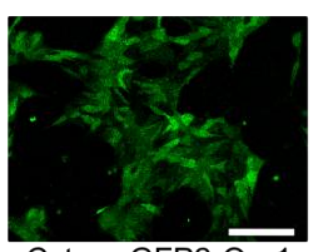

Cyto-roGFP2-Orp1

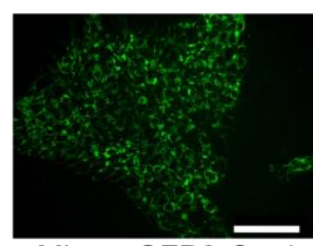

Mito-roGFP2-Orp1
D

\begin{tabular}{|l|l|c|}
\hline \multicolumn{1}{|c|}{ iPSC line } & $\begin{array}{l}\text { Cyto-roGFP2- } \\
\text { Orp1 }\end{array}$ & $\begin{array}{l}\text { Mito-roGFP2- } \\
\text { Orp1 }\end{array}$ \\
\hline K7-4Lf & 5 clones & 3 clones \\
\hline SOD1-D91A & 14 clones & 11 clones \\
\hline SOD1-G128R & 12 clones & 12 clones \\
\hline iALS & 17 clones & 11 clones \\
\hline
\end{tabular}

Fig. 2. Generation of iPSCs expressing the Cyto-roGFP2-Orp1 and Mito-roGFP2-Orp1 biosensors. A Schematic of the biosensor and transactivator for doxycycline-controllable expression inserts in the AAVS1 locus. In one allele, the homologous arms (HA-L - left, HA-R - right) flank the splice acceptor site (SA), T2A peptide, the neomycinresistance gene (in the open reading frame of $P P P 1 R 12 C$ ), and rtTA under control of the CAG promoter. In another allele, the homologous arms flank the splice acceptor site (SA), T2A peptide, the puromycin-resistance gene (in the open reading frame of $P P P 1 R 12 C$ ), and the biosensor's sequence under control of the TRE-promoter. The primers used to detect the target insertions (one - inside the insert, another - outside the left homologous arm, in the AAVSI locus) are represented as black arrows. B Representative images of live iPSCs expressing the Cyto-roGFP2-Orp1 (left) or Mito-roGFP2-Orp1 (right) biosensors $24 \mathrm{~h}$ after the addition of $2 \mathrm{mg} / \mathrm{ml}$ doxycycline in the medium. Scale bar $100 \mu \mathrm{m}$. C Representative Image of the iPSC clones analyzed for the target (two top gels) and off-target (two 
bottom gels) inserts. The arrows mark target PCR products. The clones positive for the target and negative for the off-target inserts are in frames. D List of iPSC clones obtained for each type of the biosensor and SOD1 variant.

\section{Motor neurons derived from the IPSCs with mutant SOD1 show impaired axon growth}

We utilized a previously described protocol of highly efficient motor neuron differentiation (Fig. 3a) [35] that allowed us to obtain iPSC-derived motor neurons with a characteristic morphology within 30 days. For each type of iPSC (K7-4Lf, iALS, SOD1-D91A, and SOD1-G128R), we differentiated three separate clones. All motor neurons derived from the iPSC were positively stained for ChAT, ISL1, and MNX1 and expressed mRNA of these proteins on comparable levels (Fig. 3b-c, Supplementary Fig. S5). We analyzed MN differentiation efficiency on the day 20 of differentiation by counting the $\mathrm{ISL}^{+}$cells using flow cytometry. The efficiency ranged between 89 and $95 \%$ with the $91.9 \pm 7 \% \mathrm{ISL}^{+}$cells for $\mathrm{K} 7-4 \mathrm{Lf}, 91.1 \pm 2 \%$ for SOD1-D91A, $89.3 \pm 3.8 \%$ for SOD1-G128R, and $94.7 \pm 1.5 \%$ for iALS (Fig. $3 \mathrm{~d}$ ).

To characterize MN obtained, we measured axonal length of the derived $\mathrm{MN}$ on the day 21 of differentiation in low-density culture. The mean length of the axons in SOD1-D91A and iALS MN was $90.7 \pm 42.6 \mu \mathrm{m}$ and $90.4 \pm 41.9 \mu \mathrm{m}$, respectively, which was significantly lower than in control K7-4Lf MN $(107.8 \pm 45.5 \mu \mathrm{m})$. The mean axon length in SOD1-G128R $(67 \pm 30.6 \mu \mathrm{m})$ was even lower than in SOD1-D91A and almost forty percent lower compared to the control K7-4Lf. This suggests the presence of the pathological effect of the mutations introduced in SOD1, as well as a different level of severity of these mutations (Fig. 3e).

\section{The biosensors' expression declines during differentiation}

Although the AAVS1 site is located in the intron of a transcriptionally active gene and was described previously as suitable for stable expression of transgenes [44], we have discovered that the motor neurons did not always retain detectable fluorescence level of the biosensors at the terminal stages of the differentiation, and this did not depend on the particular cell line used for the differentiation (Fig. 3f-g). Analysis of the expression level of rtTA and roGFP2 in the MN 
that lost the biosensor's signal revealed that the terminally differentiated MN expressed mRNA of the rtTA at the same level as the corresponding IPSCs from which they were obtained, while the expression of the biosensor's roGFP2 was decreased by two orders, suggesting that the biosensor's promoter was selectively inhibited (Fig. 3h). It is known that the differentiation process is accompanied by chromatin remodeling [45]. Since the rtTA expression is constitutive, while biosensors require tetracycline-derivatives to activate transcription, we suggested that the active state of the promoter during differentiation prevents its inhibition. We have been supplementing the differentiation medium with doxycycline every other day from the first day of differentiation to keep the biosensor's promoter in an active state. This resulted in a more stable expression of the biosensors' mRNA on a level comparable to the IPSC (Fig. 3h) as well as in a high level of the fluorescence intensity. 
bioRxiv preprint doi: https://doi.org/10.1101/2021.09.13.459724; this version posted September 15, 2021. The copyright holder for this preprint (which was not certified by peer review) is the author/funder, who has granted bioRxiv a license to display the preprint in perpetuity. It is made available under aCC-BY 4.0 International license.

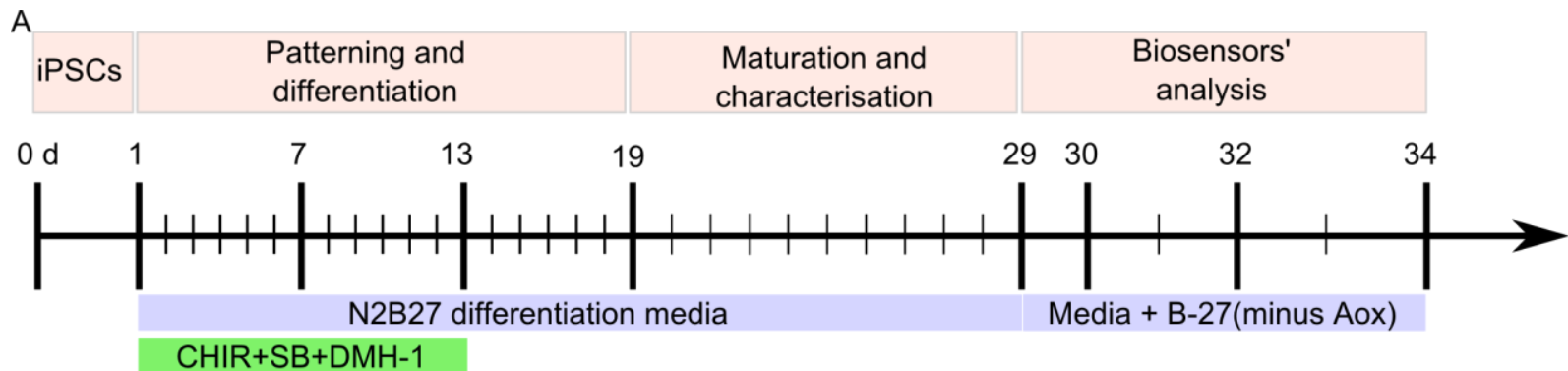

Retinoic acid

Puromorphamine

Compound $\mathrm{E}$
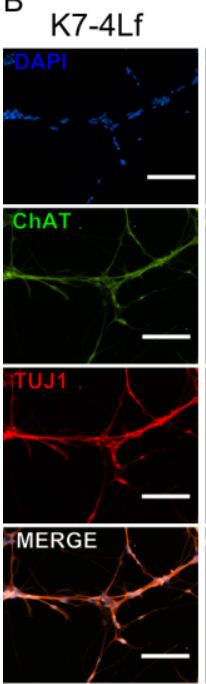

$\mathrm{D}$

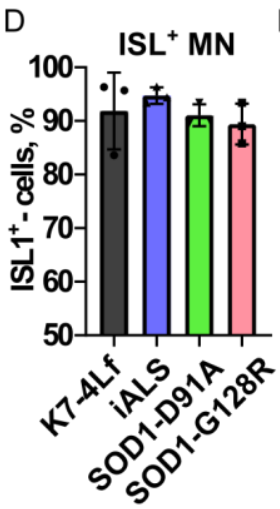

iALS

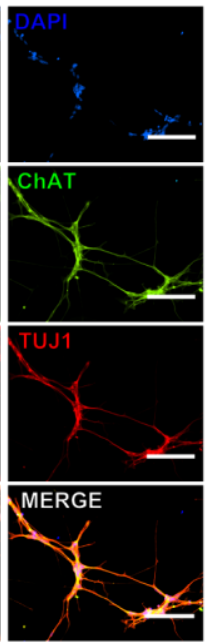

E

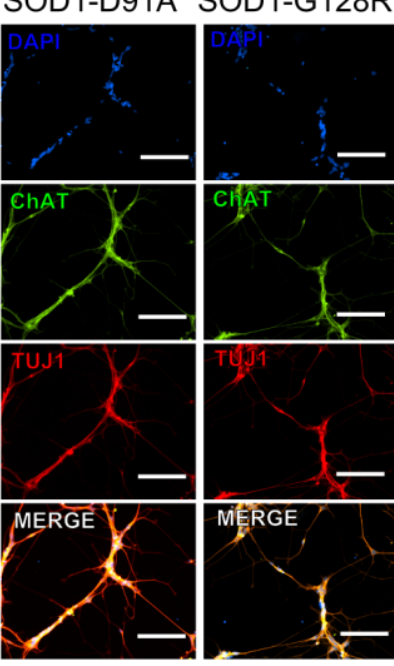

$\mathrm{F}$

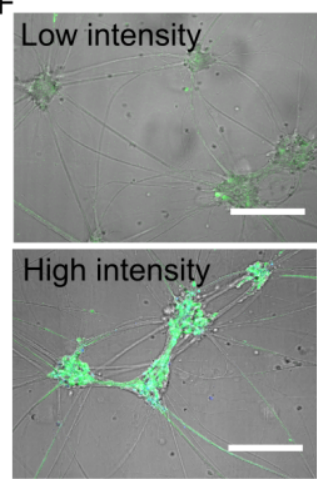

Glutamate+PDC

C

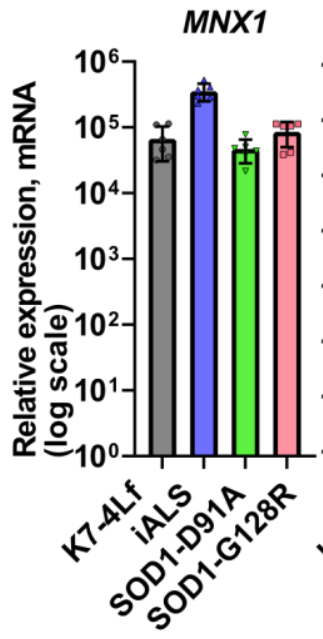

G

ISL1

ChAT
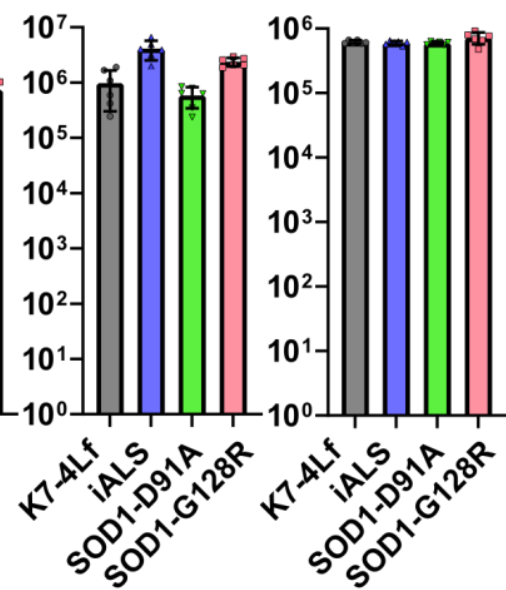

$\mathrm{H}$

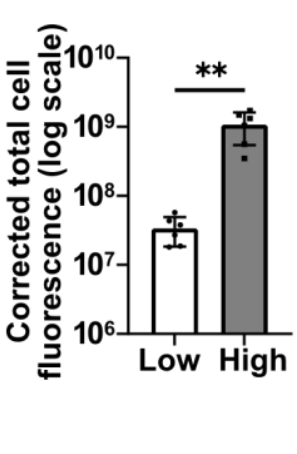

Fig. 3. Generation of iPSC-derived motor neurons expressing the Cyto-roGFP2-Orp1 and Mito-roGFP2-Orp1 biosensors. A Schematic of the differentiation protocol and a timeline for MN characterization and analysis. The main stages of the protocol are denoted above the timeline. The medium composition (basal medium and smallmolecule inhibitors) for every stage is indicated below the timeline. B Representative Images of the MN positively stained for the general neuronal marker $\beta$-III-Tubulin (TUJ1) and mature MN marker ChAT on the differentiation day 20. Nuclei are visualized with DAPI, scale bar $100 \mu \mathrm{m}$. C RT-qPCR analysis of mRNA expression of $M N X 1$, ISL1, and ChAT in K7-4Lf, iALS, SOD1-D91A, and SOD1-G128R derived MN on the differentiation day 29. Data $(\mathrm{N}=6)$ are normalized to the expression in the K7-4Lf iPSC line and presented as the mean \pm standard deviation 
(S.D.). D Quantification of flow cytometry analysis of $\mathrm{ISL}^{+}$cells performed on the differentiation day 20. Data $(\mathrm{N}=$ 3) are the mean \pm S. D. E Axon length quantification (differentiation day 21). Data $(\mathrm{N}=257,270,409,397$ for K7-

4Lf, iALS, SOD1-D91A, and SOD1-G128R, respectively) are presented as the mean \pm S.D. F Representative Images of the MN lost (top) and retained (bottom) the Cyto-roGFP2-Orp1 expression during the differentiation. Scale bar $100 \mu \mathrm{m}$. G Quantification of the Cyto-roGFP2-Orp1 fluorescence intensity in MN lost and retained the Cyto-roGFP2-Orp1 expression. Data $(\mathrm{N}=6)$ are the mean \pm S.D. H RT-qPCR analysis of the transactivator (rtTA) and biosensors (roGFP2) mRNA expression $48 \mathrm{~h}$ after $2 \mu \mathrm{g} / \mathrm{ml}$ doxycycline addition. $\mathrm{MN}+\mathrm{DOX}-\mathrm{MN}$ differentiated in the absence of doxycycline, MN + DOX (regularly) - MN differentiated with regular doxycycline supplementation, iPSCs + DOX - iPSCs expressing biosensor. Data $(\mathrm{N}=6)$ are the mean \pm S.D. $* * \mathrm{p}<0.01, * * * * \mathrm{p}$ $<0.0001$ with the Bonferroni correction for multiple comparisons where necessary.

\section{Target insertion of the genetically encoded biosensors of $\mathrm{H}_{2} \mathrm{O}_{2}$ in the $A A V S 1$ locus does not}

\section{affect their basic properties}

The main principle of the ratiometric $\mathrm{H}_{2} \mathrm{O}_{2}$ biosensors is based on the roGFP2 ability to change its fluorescent properties. Two cysteine residues positioned close to the roGFP2 chromophore form a disulfide bond under oxidation, leading to structural changes that influence the protein fluorescence [46]. Thus, the reduced roGFP2 (roGFP2red) have excitation maximum at $490 \mathrm{~nm}$, the oxidized (roGFP2ox) - at $400 \mathrm{~nm}$, with the emission maximum at $510 \mathrm{~nm}$ for both forms. It is possible to obtain a signal from each form separately by exciting the biosensor at two wavelengths; the intensity of these signals, in turn, allows estimating relative oxidation of the roGFP2, i.e. the proportion of the oxidized molecules in the cytoplasm (for Cyto-roGFP2-Orp1) or mitochondria (for Mito-roGFP2-Orp1). Fusion of the roGFP2 to the thiol peroxidase Orp1 creates a redox relay in which $\mathrm{H}_{2} \mathrm{O}_{2}$ specifically oxidizes Orp1, and then this state is passed to the roGFP2, with subsequent Orp1 reduction (Fig. 4a) [46]. Since the reaction is reversible, the biosensors reflect not only the steady-state level of $\mathrm{H}_{2} \mathrm{O}_{2}$ in the cell but also dynamic changes in $\mathrm{H}_{2} \mathrm{O}_{2}$ caused by certain events.

For the ratiometric $\mathrm{H}_{2} \mathrm{O}_{2}$ biosensors, dynamic range - the difference between the fully oxidized and fully reduced biosensor - reflects the scale of the signals that can be distinguished in the 
experiment (Fig. 4b), and according to the literature, this number varies from 3 to 8 [23,36,47].

We measured the dynamic range of the Cyto-roGFP2-Orp1 and Mito-roGFP2-Orp1 biosensors in every MN sample used in the work (Fig. 4c). To do that, we added to the cells either oxidizing agent diamide or reducing agent DTT. Afterward we recorded changes in the biosensors' signal in real-time. The biosensors quickly reacted to the addition of the oxidizing and reducing agents, reaching the plateau 8-10 minutes after the chemicals were added (Fig. 4d-e). The calculated dynamic range was $4 \pm 0.33$ for Cyto-roGFP2-Orp1 and 3.3 \pm 0.61 for Mito-roGFP2-Orp1 and stayed within acceptable values.

A

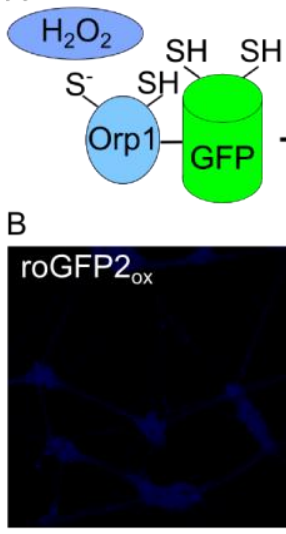

Intensity roGFP2ox

D

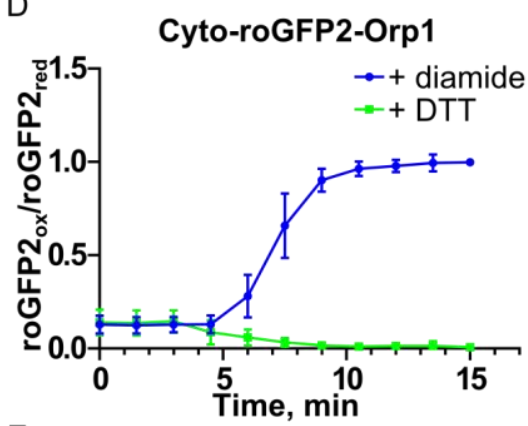

E

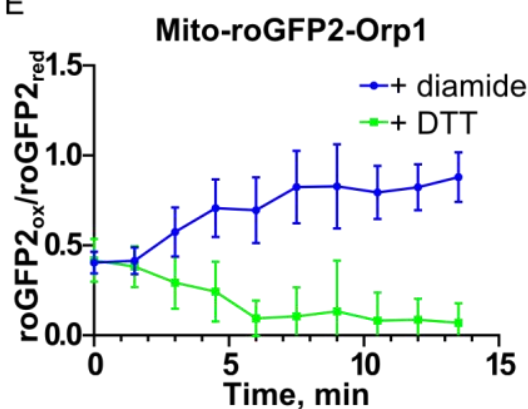

Start

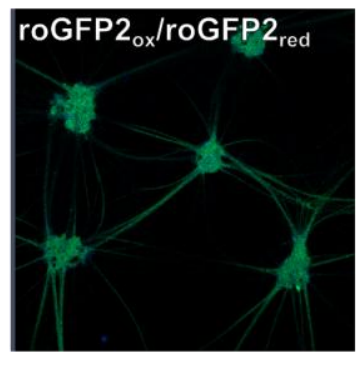

Start

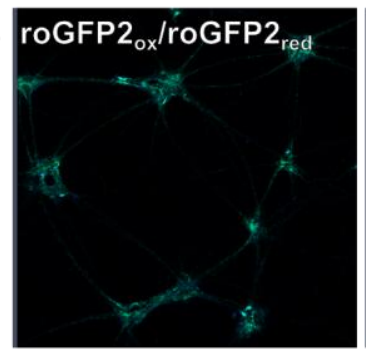

C

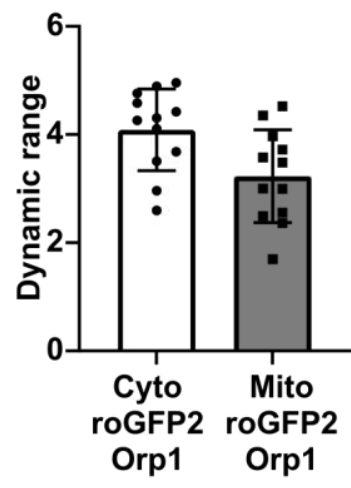

Fig. 4. Evaluation of the Cyto-roGFP2-Orp1 and Mito-roGFP2-Orp1 functionality in iPSC-derived motor neurons.

A Interaction of roGFP2-Orp1 with $\mathrm{H}_{2} \mathrm{O}_{2}$ (According to Morgan et al. [36]). The Image demonstrates how $\mathrm{H}_{2} \mathrm{O}_{2}$ 
triggers oxidation of the roGFP2 via Orp1 thiol peroxidase. B Fluorescence spectra of roGFP2 (top) in the fully reduced (roGFP $2_{\text {red }}$ ) or fully oxidized (roGFP $2_{\text {ox }}$ ) states with the representative images of MN obtained by excitation at the $405 \mathrm{~nm}$ (blue pseudocolor) and $488 \mathrm{~nm}$ (green pseudocolor) wavelengths; the equation for calculation of the relative biosensor's oxidation (bottom). C Dynamic range of the Cyto-roGFP2-Orp1 and Mito-roGFP2-Orp1 biosensors expressed in MN. Data $(\mathrm{N}=12)$ are the mean \pm standard deviation (S.D.). The reaction of the CytoroGFP2-Orp1-expressing (D) and Mito-roGFP2-Orp1-expressing (E) MN to the diamide and DTT addition with the representative images of the intact $\mathrm{MN}$ (Start), fully reduced (+ DTT) and fully oxidized (+ diamide) MN. Data (N = 9) are normalized to the values of the maximum oxidation and reduction and presented as the mean \pm S.D. The Images present an overlap of the $405 \mathrm{~nm}$ (oxidized roGFP2) and $488 \mathrm{~nm}$ (reduced roGFP2) channels colored in blue and green pseudocolors, respectively.

\section{B-27 supplement deprivation affects mitochondrial level of $\mathrm{H}_{2} \mathrm{O}_{2}$ regardless of the genotype}

The basal level of $\mathrm{H}_{2} \mathrm{O}_{2}$ reflects redox balance and general condition of the cell. To obtain information about the redox state of the $\mathrm{MN}$, we performed live imaging on the differentiation day 29. We did not observe any differences between K7-4Lf and SOD1-D91A MN: relative oxidation level of the cytoplasm and mitochondria was the same for both types of neurons (Fig. 5a). No differences were also detected between these two cellular compartments. The cytoplasmic $\mathrm{H}_{2} \mathrm{O}_{2}$ level in iALS MN was slightly lower compared to the control (K7-4Lf), but the mitochondrial $\mathrm{H}_{2} \mathrm{O}_{2}$ level was the same. We suggested that these findings are probably due to the different MN origin of iALS MN since the SOD1-D91A oxidation values did not differ from the control, although this statement requires additional research. SOD1-G128R MN, however, demonstrated a 2.7 times higher level of $\mathrm{H}_{2} \mathrm{O}_{2}$ in the cytoplasm and a 5 times higher level of the mitochondrial $\mathrm{H}_{2} \mathrm{O}_{2}$ compared to the control. Together with an overall higher level of the oxidation in both these compartments, we observed that the mitochondrial level of $\mathrm{H}_{2} \mathrm{O}_{2}$ was twice as high as the cytoplasmic. Notably, that the cytoplasmic oxidation measured at the differentiation day 20 (immature MN) was the same for all neurons, suggesting that the SOD1G128R MN dysfunction, probably caused by the SOD1 mutation, occurs only in mature MN (Fig. 5b). To correct the observed phenotype, we added a combination of neurotrophic factors 
(NTF) to the culture medium during SOD1-G128R MN maturation (differentiation days 19-29). This resulted in significant decrease in cytoplasmic oxidation to the normal level. The mitochondrial level of $\mathrm{H}_{2} \mathrm{O}_{2}$, however, was not affected by the NTF addition (Fig. 5c).

Standard culture conditions are not always beneficial for the redox studies because of the protective influence of the standard medium. We removed the B-27 supplement from the medium $24 \mathrm{~h}$ before live imaging of the $\mathrm{MN}$ and measured the cytoplasmic and mitochondrial $\mathrm{H}_{2} \mathrm{O}_{2}$ levels (Fig. 5d). We discovered that B-27 deprivation did not influence the cytoplasmic level of $\mathrm{H}_{2} \mathrm{O}_{2}$. Although $\mathrm{MN}$ cultured in the absence of B-27 demonstrated significant increase in the mitochondrial $\mathrm{H}_{2} \mathrm{O}_{2}$ level, which was, on average, 4 times higher than in the cytoplasm of the corresponding neurons. For the SOD1-G128R MN, the B-27 deprivation resulted in an elevation of both cytoplasmic and mitochondrial $\mathrm{H}_{2} \mathrm{O}_{2}$ levels to the values higher than in control $\mathrm{K} 7-4 \mathrm{Lf}$ MN (Fig 5d).

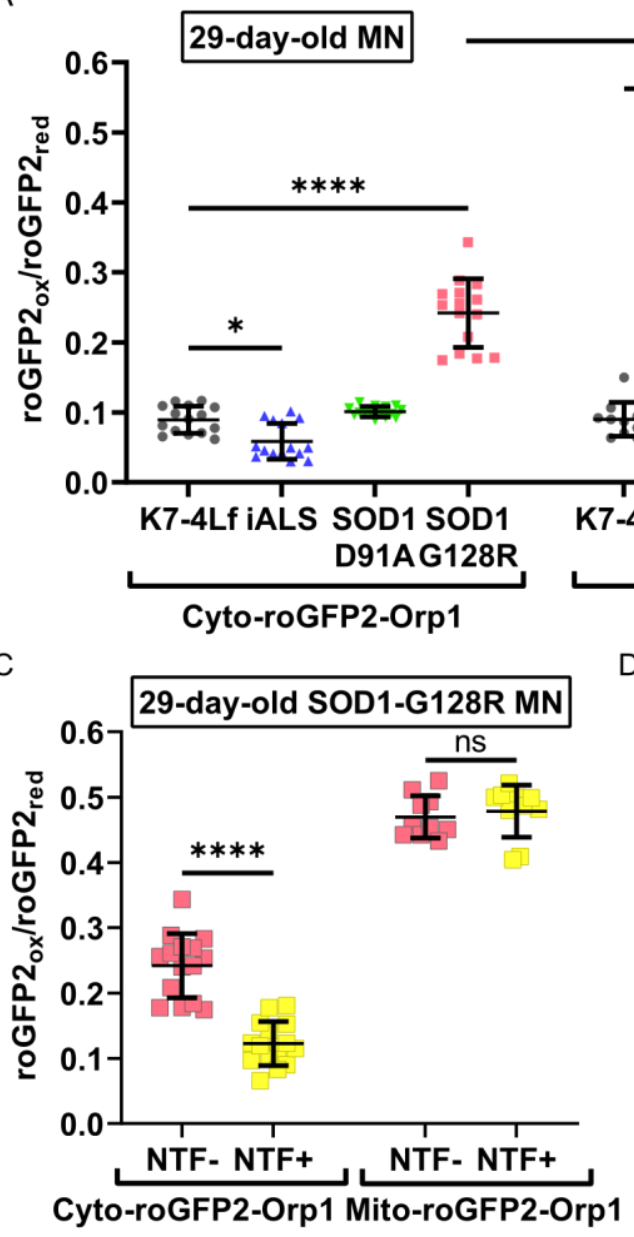

B

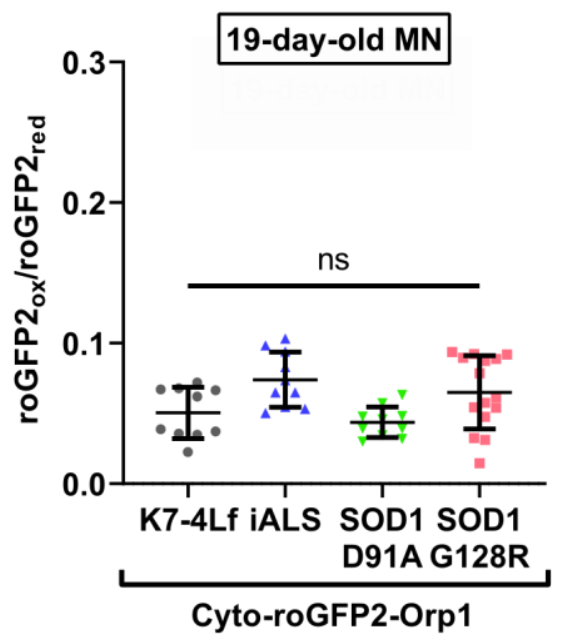

D

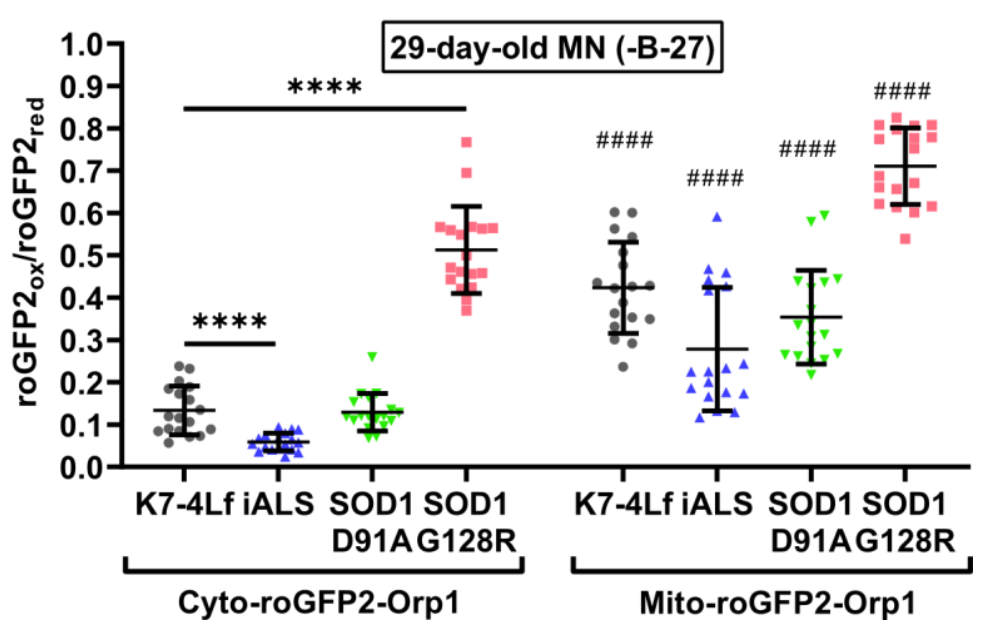


Fig. 5. Basal levels of $\mathrm{H}_{2} \mathrm{O}_{2}$ in the cytoplasm and mitochondria of the Cyto-roGFP2-Orp1 and Mito-roGFP2-Orp1 expressing $\mathrm{MN}$ are affected by culture conditions, genotype, and degree of maturation. A Basal levels of $\mathrm{H}_{2} \mathrm{O}_{2}$ in the cytoplasm and mitochondria of the Cyto-roGFP2-Orp1 and Mito-roGFP2-Orp1 expressing MN measured on the differentiation day 29. B A basal level of $\mathrm{H}_{2} \mathrm{O}_{2}$ in the cytoplasm of the Cyto-roGFP2-Orp1 expressing MN measured on the differentiation day 20. C Basal levels of $\mathrm{H}_{2} \mathrm{O}_{2}$ in the cytoplasm and mitochondria of the Cyto-roGFP2-Orp1 and Mito-roGFP2-Orp1 expressing SOD1-G128R MN measured on the differentiation day 29 depending on the presence of neurotrophic factors in the culture medium during maturation. D Basal levels of $\mathrm{H}_{2} \mathrm{O}_{2}$ in the cytoplasm and mitochondria of the Cyto-roGFP2-Orp1 and Mito-roGFP2-Orp1 expressing MN in the B-27 deprived medium measured on the differentiation day 29. Data $(\mathrm{N}=10-15,18$ for $\mathrm{B}-27$ deprived samples $)$ are the mean \pm standard deviation. $* * * \mathrm{p}<0.001, * * * * \mathrm{p}<0.0001$ with the Bonferroni correction for multiple comparisons where necessary; \#\#\# and \#\#\#\# denote $\mathrm{p}<0.001$ and $\mathrm{p}<0.0001$, respectively, in comparisons between the mitochondrial and cytoplasmic level of $\mathrm{H}_{2} \mathrm{O}_{2}$ of the same $\mathrm{MN}$ sample. The same datasets of the cytoplasmic and mitochondrial $\mathrm{H}_{2} \mathrm{O}_{2}$ levels in SOD1-G128R (-NTF) were used on the images $\mathbf{A}$ and $\mathbf{C}$.

\section{Antioxidants removal from the medium induces $\mathrm{H}_{2} \mathrm{O}_{2}$ accumulation in the cytoplasm of}

\section{motor neurons}

Removal of the antioxidants from the medium forces $\mathrm{MN}$ to rely on endogenous antioxidant systems for the ROS neutralization. We cultured motor neurons in the neuronal maintenance medium (supplemented with B-27 w/o antioxidants) for three days in addition to the differentiation protocol and performed live imaging of the MN (Fig. 6a-b). We observed an increase in the level of cytoplasmic $\mathrm{H}_{2} \mathrm{O}_{2}$ in the $\mathrm{MN}$ by $1.5-2.5$ times. The mitochondrial $\mathrm{H}_{2} \mathrm{O}_{2}$ level did not change for control MN, iALS MN, and SOD1-D91A MN. However, together with increased cytoplasmic oxidation, SOD1-G128R MN demonstrated a rise in mitochondrial oxidation. This alteration in the $\mathrm{H}_{2} \mathrm{O}_{2}$ level was accompanied by visible changes in the SOD1G128R MN morphology with the axon attrition and cytoplasmic vacuolization (Fig. 6c). Culturing of SOD1-G128R MN with the NTF reduced oxidation of the cytoplasm, but it did not manage to keep it to the normal level (Fig. 6d). The mitochondrial level of $\mathrm{H}_{2} \mathrm{O}_{2}$, again, was unaffected by the NTF addition. 
A

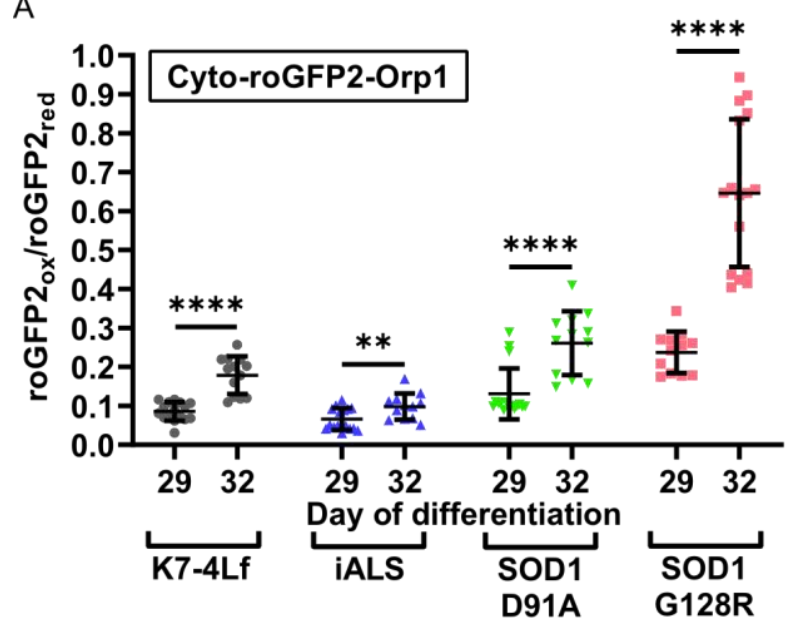

C

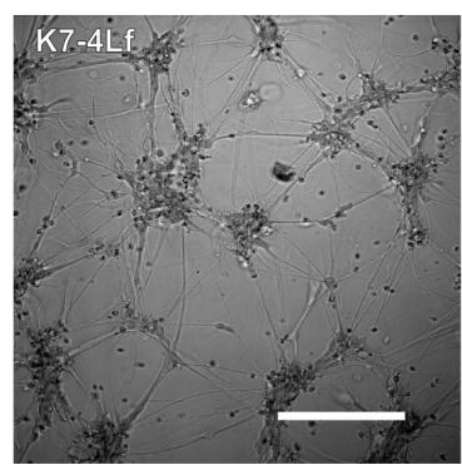

B

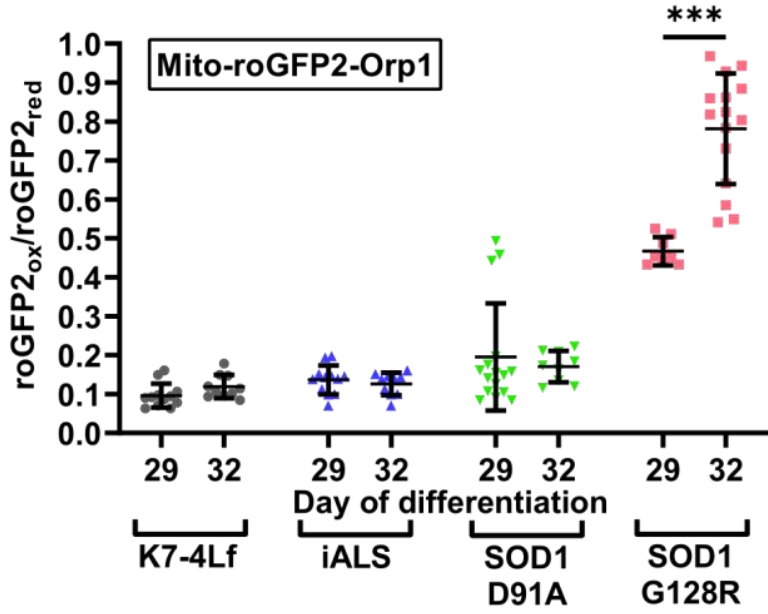

D

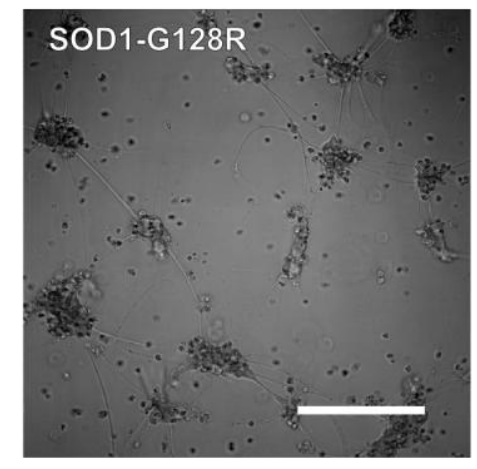

32-day-old SOD1-G128R MN

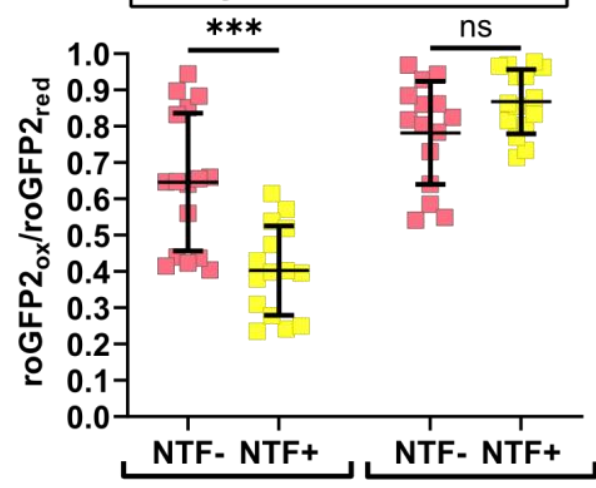

Cyto-roGFP2-Orp1 Mito-roGFP2-Orp1

Fig. 6. Effect of the antioxidant deprivation on the $\mathrm{H}_{2} \mathrm{O}_{2}$ level in the cytoplasm and mitochondria of motor neurons. A basal level of $\mathrm{H}_{2} \mathrm{O}_{2}$ in the cytoplasm (A) and mitochondria (B) of $\mathrm{MN}$ measured on the differentiation days 29 and 32. C Representative Images of the K7-4Lf and SOD1-G128R MN morphology in the antioxidant-deprived medium (differentiation day 32, DIC contrast), scale bar $200 \mu \mathrm{m}$. D Basal levels of $\mathrm{H}_{2} \mathrm{O}_{2}$ in the cytoplasm and mitochondria of SOD1-G128R MN measured on the differentiation day 32 depending on the presence of the neurotrophic factors in the culture medium. Data $(\mathrm{N}=10-16)$ are the mean \pm standard deviation. $\mathrm{ns}-$ non significant, $* * \mathrm{p}<0.01, * * * \mathrm{p}<$ 0.001 , **** $\mathrm{p}<0.0001$. The same datasets of cytoplasmic and mitochondrial $\mathrm{H}_{2} \mathrm{O}_{2}$ levels in SOD1-G128R (-NTF) were used on the images $\mathbf{A}, \mathbf{B}$, and $\mathbf{C}$. The datasets used to present the cytoplasmic and mitochondrial oxidation on the differentiation day 29 for K7-4Lf, iALS, SOD1-D91A, and SOD1-G128R MN on the images $\mathbf{A}$ and $\mathbf{B}$ are the same as in Fig. 5A. 


\section{Cyto-roGFP2-Orp1 biosensor expressed in motor neurons reflects the kinetics of $\mathrm{H}_{2} \mathrm{O}_{2}$}

\section{neutralization}

$\mathrm{H}_{2} \mathrm{O}_{2}$ is a target molecule for the Cyto-roGFP2-Orp1 and Mito-roGFP2-Orp1 biosensors, and it directly affects the sensors' properties and induces specific signals. At the same time, the addition of $\mathrm{H}_{2} \mathrm{O}_{2}$ to the cultured cells is a known method for stress induction [48]. The main advantage of the GE biosensors is that they allow measuring the $\mathrm{H}_{2} \mathrm{O}_{2}$ level in real-time, which is important for the study of dynamic cellular reactions. Thus, it is possible to record the process of $\mathrm{H}_{2} \mathrm{O}_{2}$ utilization by the cell. But the addition of deliberately lethal for the cells concentrations of $\mathrm{H}_{2} \mathrm{O}_{2}$ hinders the opportunity to compare the cellular response and its features caused by the genotype of interest. First, to find a maximum amount of hydrogen peroxide molecules that cultured $\mathrm{MN}$ can neutralize, staying alive, we tried a range of $\mathrm{H}_{2} \mathrm{O}_{2}$ concentrations. We found that $\mathrm{MN}$ was tolerant to $10 \mu \mathrm{M} \mathrm{H}_{2} \mathrm{O}_{2}$, but $25 \mu \mathrm{M} \mathrm{H}_{2} \mathrm{O}_{2}$ induced death of the cells (data not shown). Second, we tested whether the standard culture conditions distort the cellular response to the $\mathrm{H}_{2} \mathrm{O}_{2}$. We performed a live recording of the Cyto-roGFP2-Orp1-expressing MN reaction to the addition of $10 \mu \mathrm{M} \mathrm{H}_{2} \mathrm{O}_{2}$. These $\mathrm{MN}$ were either cultured in the standard NDM or starved in the B-27-deprived medium before the measurement (Fig. 7a). The overall reaction of the cells was similar regardless of the culture conditions; the MN expressing Cyto-roGFP2-Orp1 demonstrated oxidation followed by slow reduction, reflecting the change in the cytoplasmic $\mathrm{H}_{2} \mathrm{O}_{2}$ level. However, the reaction of $\mathrm{MN}$ cultured in the B-27-deprived medium before the experiment was more prominent. We detected a higher value of the maximum biosensor oxidation and faster reduction compared to the non-starved MN (Fig. b-c). Since the cellular reaction was affected by the components present in the standard medium, we conducted further measurements of the dynamic response on the cells that were starved before the experiment. Using the parameters of the experiment established earlier, we recorded the reaction of $\mathrm{MN}$ expressing the Mito-roGFP2-Orp1 biosensor to $10 \mu \mathrm{M} \mathrm{H}_{2} \mathrm{O}_{2}$ in real-time. We did not detect any 
changes in response to the exogenous $\mathrm{H}_{2} \mathrm{O}_{2}$; an oxidation value for the Mito-roGFP2-Orp1 biosensor remained constant during imaging, suggesting that the mitochondrial $\mathrm{H}_{2} \mathrm{O}_{2}$ level was also constant (Fig. 7d). The addition of $\mathrm{H}_{2} \mathrm{O}_{2}$ in higher concentrations $(25 \mu \mathrm{M}$ and $50 \mu \mathrm{M})$ induced mitochondrial oxidation but, as it was found earlier, damaged the neurons. This fact forced us to abandon the measurement of the dynamic response for the Mito-roGFP2-Orp1 sensor in further experiments (Fig. 7e).

Next, we analyzed how the mutations introduced in SOD1 affected neuronal reaction to the exogenous $\mathrm{H}_{2} \mathrm{O}_{2}$. We did not find any differences in the $\mathrm{H}_{2} \mathrm{O}_{2}$ utilization in the cytoplasm of Cyto-roGFP2-Orp1-expressing SOD1-D91A, iALS, and control (K7-4Lf) MN. The value of the maximum biosensor oxidation was similar between the cells, as well as the rate of recovery after the addition of $\mathrm{H}_{2} \mathrm{O}_{2}$ (Fig. 7f-h). Analysis of the response of the SOD1-G128R MN to the $\mathrm{H}_{2} \mathrm{O}_{2}$ revealed an aberrant reaction due to the initial high oxidation of the $\mathrm{MN}$; the level of cytoplasmic oxidation remained constantly high before and after the addition of $\mathrm{H}_{2} \mathrm{O}_{2}$ (Fig. 7f, 7i). Although culturing of the SOD1-G128R MN with the NTF slightly reduced initial oxidation of the cytoplasm, it did not significantly affect the cell reaction to the $\mathrm{H}_{2} \mathrm{O}_{2}$. The Cyto-roGFP2-Orp1expressing MN demonstrated moderate oxidation of the cytoplasm without signs of subsequent reduction (Fig. 7i). 
A
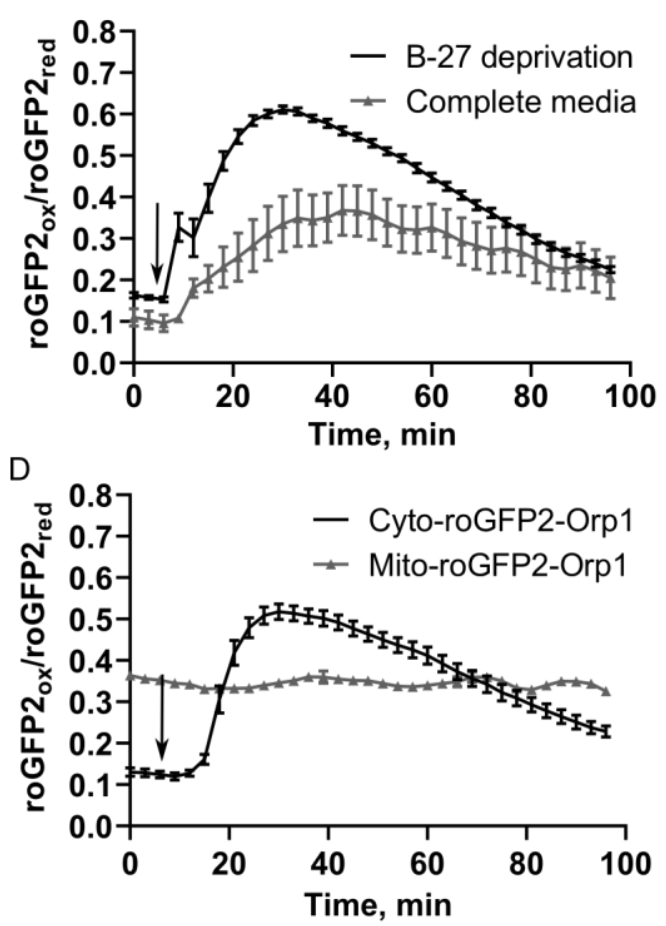

$\mathrm{F}$

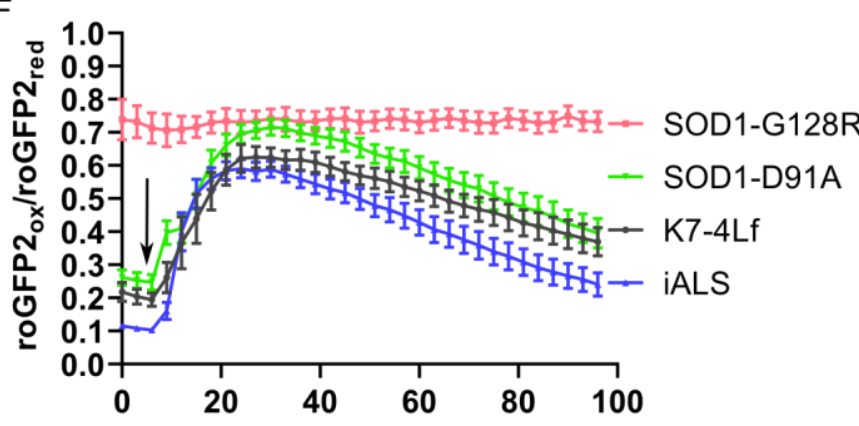

$\mathrm{H}$

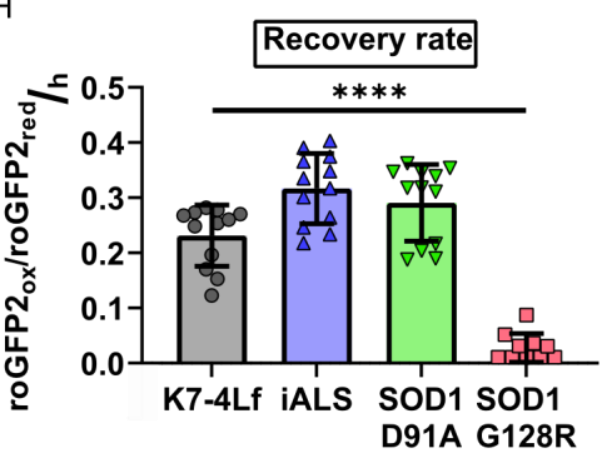

B

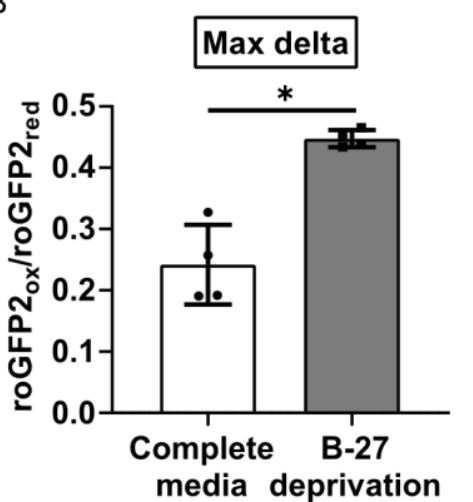

E

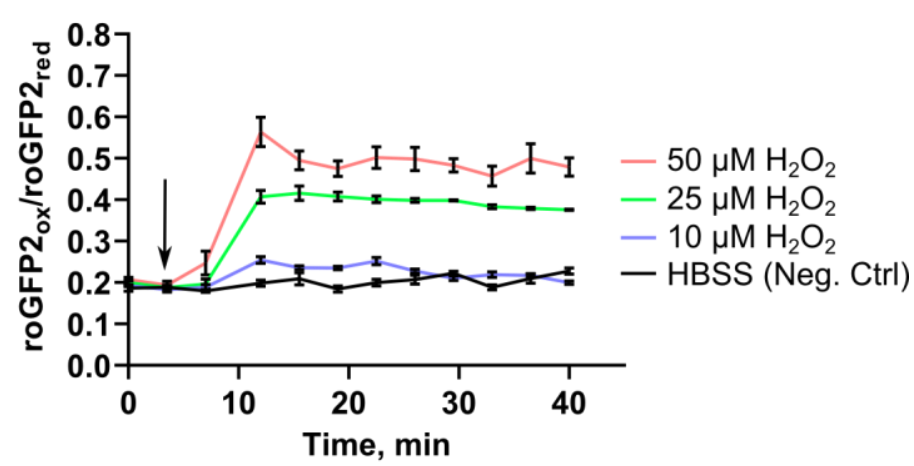

G
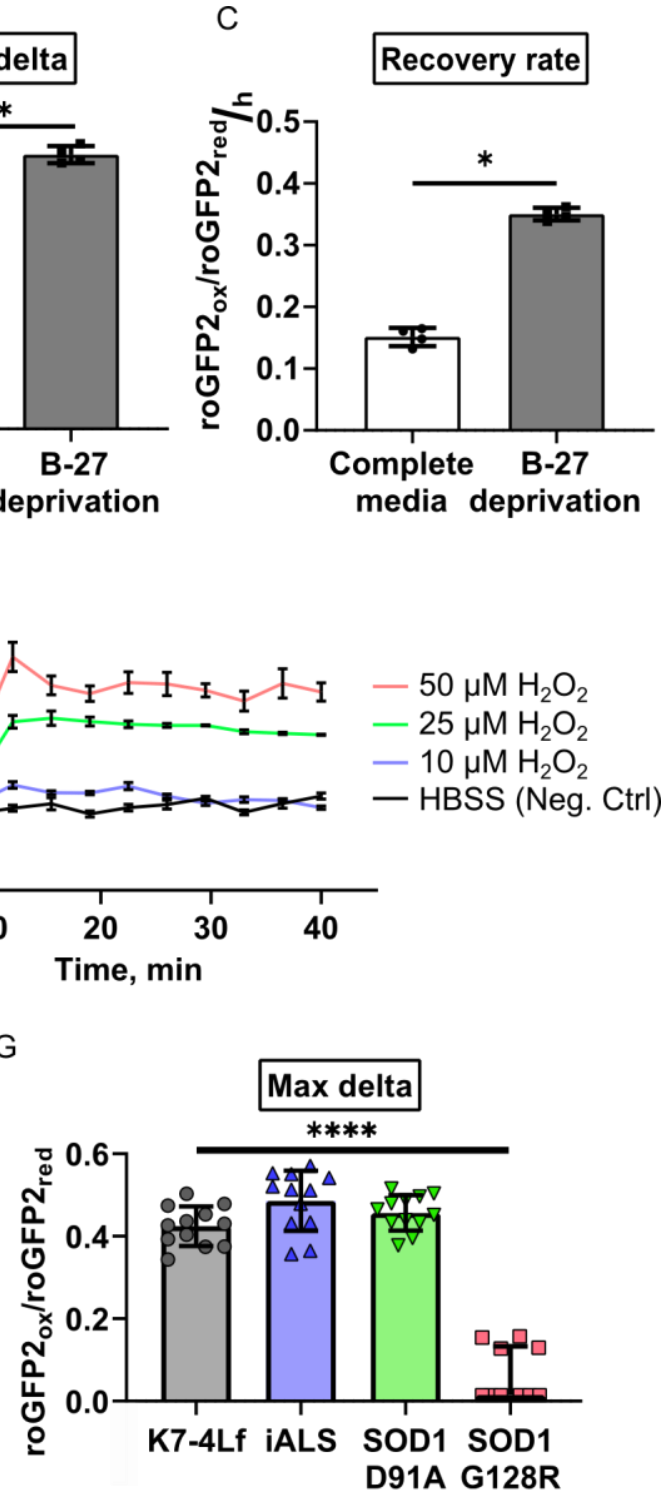

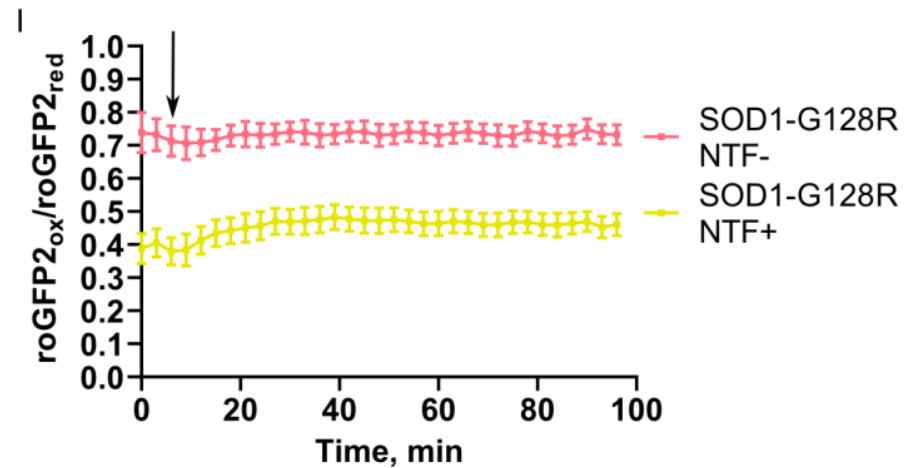

Fig. 7. Kinetics of $\mathrm{H}_{2} \mathrm{O}_{2}$ utilization in live iPSC-derived motor neurons. A Response of the K7-4Lf MN cultured in the standard medium or B-27-deprived medium to the addition of $10 \mu \mathrm{M} \mathrm{H}_{2} \mathrm{O}_{2}$. Data $(\mathrm{N}=4)$ are the mean \pm standard error of the mean (S.E.M.) Recovery rate (B) and maximum oxidation of the cytoplasm (C) of the K7-4Lf MN expressing Cyto-roGFP2-Orp1 to the $\mathrm{H}_{2} \mathrm{O}_{2}$ addition. Data $(\mathrm{N}=4)$ are the mean \pm standard deviation (S.D.) D Response of the K7-4Lf MN expressing Cyto-roGFP2-Orp1 or Mito-roGFP2-Orp1 to the addition of $10 \mu \mathrm{M} \mathrm{H}_{2} \mathrm{O}_{2}$. Data $(\mathrm{N}=4)$ are the mean \pm S.D. E Response of the K7-4Lf MN expressing Mito-roGFP2-Orp1 to the indicated 
amount of $\mathrm{H}_{2} \mathrm{O}_{2}$. Data $(\mathrm{N}=3)$ are the mean \pm S.D. F Response of the K7-4Lf, iALS, SOD1-D91A, and SOD1G128R MN expressing Cyto-roGFP2-Orp1 to the addition of $10 \mu \mathrm{M} \mathrm{H}_{2} \mathrm{O}_{2}$. Data $(\mathrm{N}=12)$ are the mean \pm S.E.M. Recovery rate $(\mathbf{G})$ and maximum oxidation of the cytoplasm $(\mathbf{H})$ of the K7-4Lf, iALS, SOD1-D91A, and SOD1G128R MN expressing Cyto-roGFP2-Orp1 after $10 \mu \mathrm{M} \mathrm{H}_{2} \mathrm{O}_{2}$ addition. Negative values in the SOD1-G128R sample were set to 0 on the graphs. Data $(\mathrm{N}=12)$ are the mean $\pm \mathrm{S}$. D. I Response of the SOD1-G128R MN expressing Cyto-roGFP2-Orp1 cultured in the standard medium or medium supplemented with neurotrophic factors to $10 \mu \mathrm{M}$ $\mathrm{H}_{2} \mathrm{O}_{2}$. The moment of the $\mathrm{H}_{2} \mathrm{O}_{2}$ addition is marked by an arrow. Data $(\mathrm{N}=4)$ are the mean \pm S.E.M. $* \mathrm{p}<0.05$, $* * * * \mathrm{p}<0.0001$. The same dataset was used in the images $\mathbf{F}$ and $\mathbf{I}$ for the representation of SOD1-G128R (-NTF) reaction to $\mathrm{H}_{2} \mathrm{O}_{2}$.

\section{Motor neurons expressing the Cyto-roGFP2-Orp1 biosensor accumulate $\mathrm{H}_{2} \mathrm{O}_{2}$ in the cytoplasm due to glutamate-induced excitotoxicity}

Glutamate excitotoxicity is one of the major mechanisms of the ALS development [49]. Excessive activation of the glutamate receptors leads to an increased $\mathrm{Ca}^{2+}$ influx, subsequent mitochondrial dysfunction, and apoptosis [50]. It is known that this process is accompanied by the increased ROS production, which connects it with the oxidative stress [51]. To test whether the Cyto-roGFP2-Orp1 and Mito-roGFP2-Orp1 biosensors can reflect redox imbalance caused by the excitotoxicity, we incubated the MN with monosodium glutamate and the glutamate reuptake inhibitor (PDC) for 5 days and measured the cytoplasmic and mitochondrial $\mathrm{H}_{2} \mathrm{O}_{2}$ levels. Since SOD1-G128R MN (both control and experimental) died shortly after the beginning of the experiment due to the reduced viability, the measurement was conducted only for K7-4Lf, SOD1-D91A, and iALS MN. We discovered that the glutamate treatment induced the accumulation of $\mathrm{H}_{2} \mathrm{O}_{2}$ in the cytoplasm, but not in the mitochondria, despite the known connection of mitochondrial dysfunction with the excitotoxicity (Fig. 8a-b). We observed mitochondrial oxidation in SOD1-D91A MN in both control and glutamate treated samples, although we were unable to determine if the oxidation was a hallmark of the SOD1 mutation or a technical artifact. The oxidation of the cytoplasm in K7-4Lf MN treated with glutamate was $28 \%$ higher, in iALS MN - by $41 \%$ higher, in SOD1-D91A MN - by $31.5 \%$ higher compared to the 
non-treated sample (Fig. 8a). We observed that iALS MN responded to the glutamate treatment more prominently than control MN. However, we did not find the same for SOD1-D91A (Fig. 8a), which makes us suggest that this effect was not caused by the SOD1 mutation.

Further, we investigated how incubation with the monosodium glutamate affected dynamics of $\mathrm{H}_{2} \mathrm{O}_{2}$ utilization in the cytoplasm of the Cyto-roGFP2-Orp1-expressing MN. We found that MN treated with glutamate had reduced recovery rate after the $\mathrm{H}_{2} \mathrm{O}_{2}$ addition compared to the nontreated sample (Fig. 8c-g). We also observed the tendency towards the slower recovery of the mutant MN (36\% slower for SOD1-D91A MN, 38\% slower for iALS MN, 23\% slower for K74Lf MN), however, it was insignificant.

A

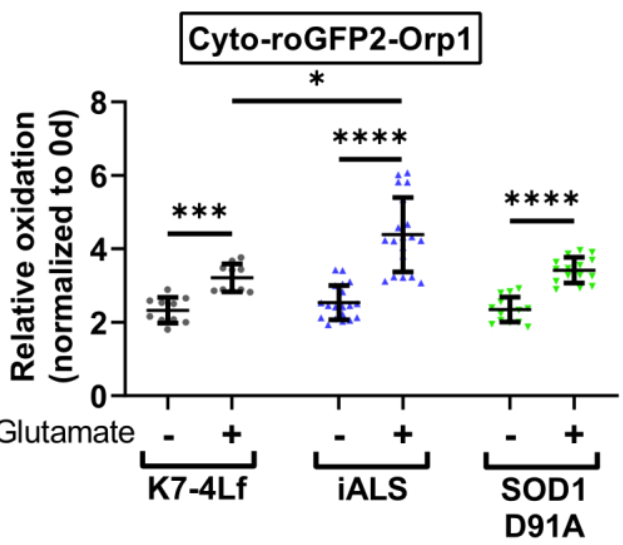

C

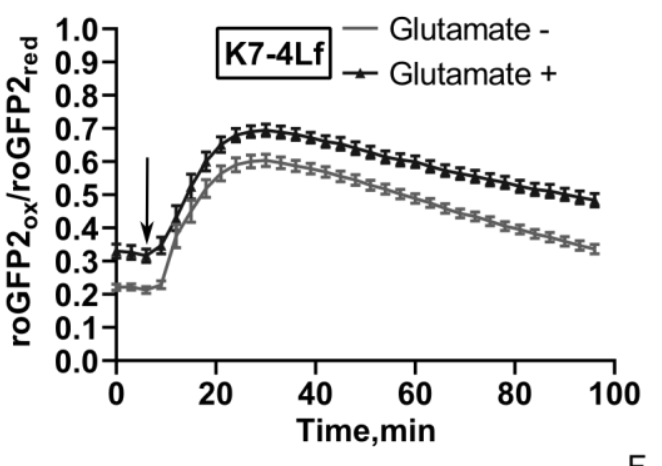

E

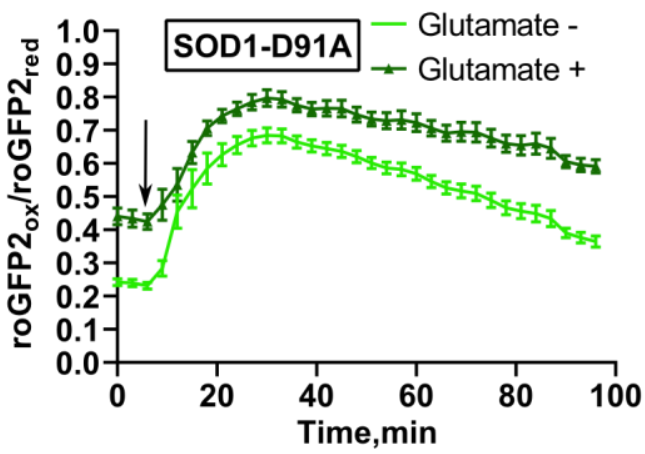

B

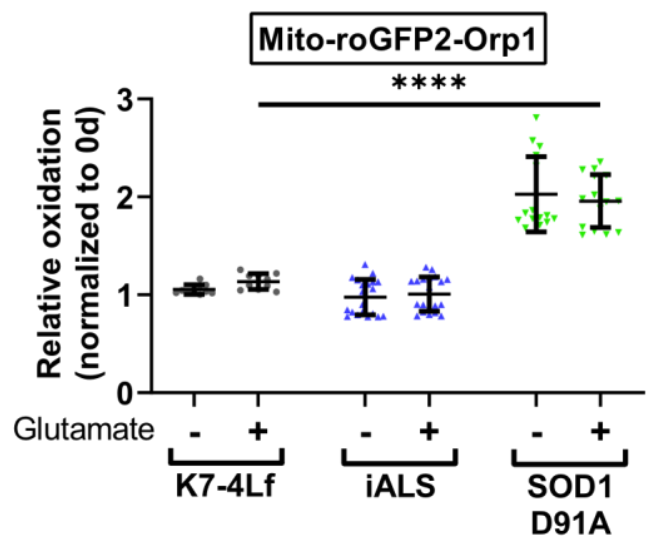

D
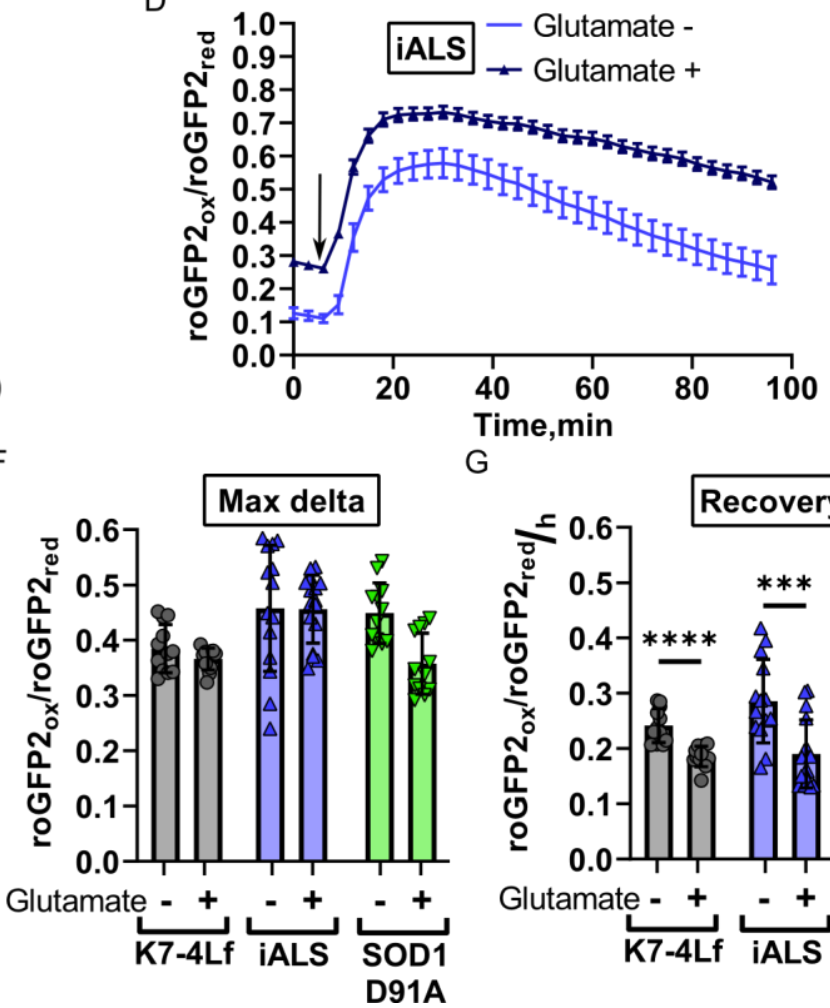

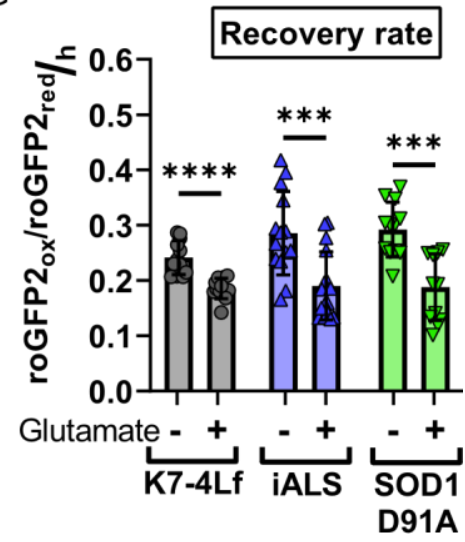


Fig. 8. The reaction of the iPSC-derived motor neurons to the excitotoxicity. A basal level of $\mathrm{H}_{2} \mathrm{O}_{2}$ in the cytoplasm (A) and mitochondria (B) of MN after 5-days incubation with glutamate. Data $(\mathrm{N}=10-15)$ are normalized to the basal $\mathrm{H}_{2} \mathrm{O}_{2}$ level measured before the addition of glutamate and presented as the mean \pm standard deviation (S.D.). The response of K7-4Lf (C), iALS (D), and SOD1-D91A (E) MN to the addition of $10 \mu \mathrm{M} \mathrm{H}_{2} \mathrm{O}_{2}$ (marked by an arrow). The reaction of $\mathrm{MN}$ cultured with glutamate is depicted in the darker graphs. Data $(\mathrm{N}=12)$ are the mean \pm standard error of the mean (S.E.M.) Maximum oxidation of the cytoplasm (F) and recovery rate (G) of the K7-4Lf, iALS, and SOD1-D91A MN expressing Cyto-roGFP2-Orp1 after the addition of $10 \mu \mathrm{M} \mathrm{H}_{2} \mathrm{O}_{2}$. Data $(\mathrm{N}=12)$ are the mean \pm S. D. $* \mathrm{p}<0.05, * * * \mathrm{p}<0.001, * * * * \mathrm{p}<0.0001$

\section{DISCUSSION}

In the present work, we established a valid approach for studying neurodegeneration in cellbased models using a combination of methods such as CRISPR/Cas9 genome editing, genetically encoded biosensors, and iPSC differentiation. Nowadays, GE biosensors are more often applied for research of different physiological and pathological processes $[47,52,53]$ where they replaced molecular probes. In the case of redox processes, it provided information about dynamics of components of redox balance, e.g., hydrogen peroxide or GSH/GSSG ratio, in different cell compartments and tissues [23,47,54,55]. Since biosensors molecules are delivered inside the cells as plasmids for temporal expression or as viral vectors for sequence integration in the genome, the robustness of biosensors-related studies often relies on the efficiency of delivery and place of transgene integration. Here, we tried to improve the existing approach for biosensors experiments by target insertion of the cytoplasmic and mitochondrial $\mathrm{H}_{2} \mathrm{O}_{2}$ biosensors in combination with their controlled expression. We have generated a number of IPSC lines with the insertions of both the biosensor and transactivator essential for doxycycline-dependent expression, using the simultaneous delivery of CRISPR/Cas9 RNPs and donor plasmids.

We have discovered that a single copy of the biosensor is enough for producing a sufficient signal. Moreover, it allows achieving a similar dynamic range between samples, leading to more accurate data processing [56]. However, the mean dynamic range measured in the study for 
cytoplasmic and mitochondrial biosensors was comparable with the lowest numbers observed previously in other experiments [23,47]. This indicates that the dynamic range is directly connected with the biosensor's expression level, and more powerful promoters can improve this system [57]. The original design of the research suggested controllable induction of biosensors only on particular days during differentiation to avoid an excessive amount of fluorescent protein in the cell. However, we found a substantial decrease in fluorescence signal when it was induced only at the terminal stages of MN differentiation. Following measurement of mRNA expression confirmed a significant drop of the expression level of the biosensor, but not transactivator suggesting that maintaining the promoter in an active state prevents it from silencing [58]. Regular supplementation of doxycycline during differentiation helped to sustain a high level of the biosensors' expression in MN. However, we still observed clones that partially lost their fluorescence and became mosaic, as well as clones with generally low fluorescence intensity (data not shown). All these suggest that chromatin remodeling occurring during differentiation affects transgenes expression in the AAVS1 locus and sets a restriction on the type of transgenes that can be inserted into AAVS1. Although this problem can be overcome for biosensors [52], transgenes that are not supposed to be constantly active, such as cell-specific reporters, will not work well due to their promoter silencing $[59,60]$. This fact underlines the necessity to discover new safe harbors in the human genome and test them not only in terms of safety but the ability to sustain a desirable level of transgene expression.

Using Cyto-roGFP2-Orp1 and Mito-roGFP2-Orp1 biosensors, we observed how MN genotype, culture conditions, and cell age affected the basal level of $\mathrm{H}_{2} \mathrm{O}_{2}$ in cytoplasm and mitochondria of the $\mathrm{MN}$ and their reaction to exogenous $\mathrm{H}_{2} \mathrm{O}_{2}$. We found that starvation, induced by the B-27 supplement removal from the medium, increased the mitochondrial level of $\mathrm{H}_{2} \mathrm{O}_{2}$ but generally did not affect the cytoplasmic level of $\mathrm{H}_{2} \mathrm{O}_{2}$ unless a severe genetic mutation was present (See further). Apparently, the rich composition of the standard NDM provides the environment that protects $\mathrm{MN}$ from various stressors coming from both outside and inside the culture system. 
Starvation caused by B-27 deprivation induced moderate stress that served as a protection mechanism working through mitochondrial ROS as an activator of autophagy [61-63]. The lack of influence on the cytoplasmic level of $\mathrm{H}_{2} \mathrm{O}_{2}$ in normally functioning neurons suggests that this is a part of a natural response. Although the basal level of $\mathrm{H}_{2} \mathrm{O}_{2}$ in the cytoplasm was not affected by the starvation, $\mathrm{MN}$ reacted differently to the addition of $\mathrm{H}_{2} \mathrm{O}_{2}$ in the medium; starved MN demonstrated a higher amplitude of oxidation in response to $\mathrm{H}_{2} \mathrm{O}_{2}$ and a higher rate of recovery. B-27 deprivation made $\mathrm{MN}$ more susceptible to extra oxidation (thus, the higher amplitude of oxidation) due to the absence of protective antioxidants. At the same time, mobilization of the internal antioxidant system caused by starvation resulted in a more active reaction to the exogenous stress factors. It is unclear how long $\mathrm{MN}$ should be cultured in the B27 deprived medium before the stress becomes decompensated and whether it will help to distinguish MN with different genotypes. Some authors use deficit media on astrocyte-neuron cultures, and probably this approach can be applied for MN monocultures as well $[35,64]$.

Differences between Cyto-roGFP2-Orp1 and Mito-roGFP2-Orp1 reactions to starvation reflect relative independence of the mitochondrial and cytoplasmic antioxidant systems. Additionally, this fact is confirmed by the different reactions of Cyto-roGFP2-Orp1 and Mito-roGFP2-Orp1 to exogenous $\mathrm{H}_{2} \mathrm{O}_{2}$. The absence of significant changes in the signal of the Mito-roGFP2-Orp1 sensor and the presence of a normal reaction of the Cyto-roGFP2-Orp1 sensor in response to 10 $\mu \mathrm{M} \mathrm{H} \mathrm{H}_{2} \mathrm{O}_{2}$ suggests that most exogenous $\mathrm{H}_{2} \mathrm{O}_{2}$ molecules are neutralized by the cytoplasmic antioxidant system and are not transferred in mitochondria. The reaction that only appears in response to lethal amounts of $\mathrm{H}_{2} \mathrm{O}_{2}$ suggests that exceeding of hydrogen peroxide level in the cytoplasm above the values that it can neutralize leads to a transfer of $\mathrm{H}_{2} \mathrm{O}_{2}$ molecules in mitochondria and apoptosis induction [50]. This effect has not been shown earlier, probably because previous studies did not focus on cell recovery and used large amounts of $\mathrm{H}_{2} \mathrm{O}_{2}$ for biosensor oxidation [23]. Possible insufficiently high dynamic range also can be the reason since it does not allow detecting small changes in $\mathrm{H}_{2} \mathrm{O}_{2}$ concentration. This also can explain the lack of 
mitochondria response to the glutamate: the power of the induced stress was not enough to provoke detectable changes in mitochondrial $\mathrm{H}_{2} \mathrm{O}_{2}$, but it didn't mean that mitochondria were not affected. We believe that the application of more advanced versions of $\mathrm{H}_{2} \mathrm{O}_{2}$ biosensors with higher sensitivity and resolution in the future may be beneficial in such a study [65].

Correction of the mutation is more commonly used for to generate isogenic cell models $[66,67]$. In this work, we did not aim to discover the effect of particular SOD1 mutations but rather to test how the approaches used here can be applied for cell models. We tried to introduce the mutations in different parts of the SOD1 gene, leading to the D91A and G128R substitutions in the polypeptide [40,41]. And since no homozygous variants have been found, we selected the lines that have one allele with the target mutation and the other with large deletion (SOD1-D91A) or premature termination codon (SOD1-G128R) to maximize potential damage and make pathological phenotype more perceptible.

We found that $\mathrm{MN}$ derived from the SOD1-G128R iPSC line demonstrated significant differences in functioning that were detected by the biosensors. The most apparent is the higher level of cytoplasmic and mitochondrial oxidation that was found in the MN. Moreover, SOD1G128R MN reacted more prominent than others to the stress tests such as starvation, induced by B-27 deprivation and culturing in the antioxidant-deprived medium. Notably, immature SOD1G128R MN did not show differences in cytoplasmic $\mathrm{H}_{2} \mathrm{O}_{2}$, suggesting that the pathological phenotype requires time to develop, as it happens in ALS patients, who usually develop the symptoms after a certain age [68]. Neurotrophic factors affected cytoplasmic but not mitochondrial $\mathrm{H}_{2} \mathrm{O}_{2}$ level, although it became less apparent in the antioxidant-deprived medium. This suggests that SOD1-G128R MN functioning only relies on a highly protective culture system, and these MN are unable to sustain their viability without additional help. An elevated level of mitochondrial $\mathrm{H}_{2} \mathrm{O}_{2}$ regardless of the NTF addition shows the presence of unresolved mitochondrial malfunction that could not be corrected at that point. Presumably, mitochondrial dysfunction of SOD1-G128R MN resulting from the presence of mutant SOD1 in the cell leads 
to the redox imbalance and $\mathrm{H}_{2} \mathrm{O}_{2}$ accumulation inside the mitochondria at first [69]. Then, when the $\mathrm{H}_{2} \mathrm{O}_{2}$ level reaches the limit of buffering capacity in mitochondria, $\mathrm{H}_{2} \mathrm{O}_{2}$ enters the cytoplasm, probably due to the mitochondria death and fragmentation, initiating apoptosis. The presence of mitochondrial dysfunction also explains the slower axon growth since the lack of energy due to the dysfunction forces the cell to focus on survival rather than rebuilding the cytoskeleton [70].

Despite the significant, albeit not so prominent, decrease in axonal growth rate observed for SOD1-D91A and iALS MNs, cytoplasmic and mitochondrial $\mathrm{H}_{2} \mathrm{O}_{2}$ levels did not demonstrate significant differences associated with SOD1 mutation from the control. Patient-specific iALS MN demonstrated even lower $\mathrm{H}_{2} \mathrm{O}_{2}$ levels in some measurements, but the data was inconsistent and did not reproduce in SOD1-D91A MN, suggesting that this effect was related to the line specificity since iALS has a different origin. This data highlights the necessity to apply isogenic iPSC since such differences can lead to wrong conclusions. Glutamate-induced excitotoxicity intended to uncover mutant MN malfunction did not help as well. Although glutamate treated neurons demonstrated accumulation of $\mathrm{H}_{2} \mathrm{O}_{2}$ in the cytoplasm and slower recovery rate after $\mathrm{H}_{2} \mathrm{O}_{2}$ addition, no differences between mutant and control $\mathrm{MN}$ were observed, suggesting that the power of stress or the degree of $\mathrm{MN}$ maturation were insufficient for the pathological phenotype to reveal. Nevertheless, different degree of disturbances observed in SOD1-D91A and SOD1-G128R motor neurons in the study allows concluding that these neurons recapitulate the clinical effect of such mutations in terms of different levels of severity of the symptoms.

\section{CONCLUSION}

We developed a cell-based platform that allowed us to study redox balance in the live motor neurons with the detection of basal oxidation of the cytoplasm and mitochondria and the dynamic response of the cells to the stressors. Using isogenic iPSCs, we confirmed that mutations affecting different parts of the SOD1 sequence have a different impact on motor 
neurons state in vitro as it has been observed in patients. Our work presents a new approach for the application of cell-based disease models for research and can be used to generate other similar models. We expect this approach to be expanded to include other disease-associated mutations or the biosensors of other cellular processes.

\section{ABBREVIATIONS}

AAVS1 - adeno-associated virus site 1; ALS - amyotroptic lateral sclerosis; ARMS amplification-refractory mutation system; CRISPR/Cas9 - clustered regularly interspaced short palindromic repeats/ CRISPR-associated; DTT - dithiothreitol; GE biosensors - genetically encoded biosensors; gRNA - guide RNA; HBSS - Hank's balanced salt solution; iPSC induced pluripotent stem cells; MN - motor neurons; NDefM - neuronal deficit medium; NDM - neuronal differentiation medium; NTF - neurotrophic factors; RNP - ribonucleoprotein; roGFP2 - reduction-oxidation sensitive green fluorescent protein 2; ROS - reactive oxygen species; rtTA - reverse tetracycline-controlled transactivator; SOD1 - superoxide dismutase 1; ssODN - single-stranded oligodeoxynucleotide.

\section{SUPPLEMENTARY INFORMATION}

Additional file 1: Table S1. List of iPSC lines used in the study.

Additional file 2: Table S2. List of primers and oligonucleotides used in the study.

Additional file 3: Table S3. List of antibodies used in the study.

Additional file 4: Supplementary Figure 1. Graphical description of live MN sample preparation for microscopy. A Schematic representation of the protocol for MN sample preparation. B 3D reconstruction of the cell layer (differentiation day 29) obtained with the protocol. Morphology of MN seeded for the maturation on the top of the Matrigel (C) or inside layer of 33\% Matrigel (D) at the indicated days of differentiation. 
Additional file 5: Supplementary Figure 2. SOD1-D91A and SOD1-G128R iPSC lines characterization. A Pluripotent features of the SOD1-D91A and SOD1-G128R iPSC lines. Immunofluorescent staining for transcriptional factors: SOX2, OCT4, NANOG, and surface antigen TRA-1-60. Nuclei are visualized with DAPI. Scale bar $100 \mu \mathrm{M}$. IPSC colony morphology (TL - transmitted light), scale bar $150 \mu \mathrm{M}$. Alkaline phosphatase (AP) staining, scale bar $100 \mu \mathrm{M}$. B RT-qPCR analysis of mRNA expression of SOX2, OCT4, and NANOG in SOD1-D91A and SOD1-G128R iPSCs. Data $(\mathrm{N}=3)$ are normalized to the parental iPSC line (K7-4Lf) and presented as the mean \pm standard deviation. C Immunofluorescent staining of the products of in vitro spontaneous differentiation for endodermal (CK18), ectodermal (NF200), and mesodermal (aSMA) markers. Nuclei are visualized with DAPI. Scale bar $100 \mu \mathrm{M}$. D Partial sequence of exon 4 of the SOD1 gene of SOD1-D91A with the corresponding amino acid sequence. WT ref - reference sequence (parental iPSC line K7-4Lf); D91A allele- allele with the c.272A $>$ C single nucleotide substitution; Del allele - allele with 105-nucleotide deletion. E. Partial sequence of exon 5 of the SOD1 gene of SOD1-G128R with the corresponding amino acid sequences. WT ref. - reference sequence (parental iPSC line K7-4Lf); G128R allele - allele with the c.382G $>\mathrm{C}$ single nucleotide substitution; Stop allele - allele with premature termination codon. F Mycoplasma contamination detection with specific primers. Neg. ctrl $-\mathrm{H}_{2} \mathrm{O}$, Pos. ctrl mycoplasma contaminated cell line. Karyotyping of the SOD1-D91A (G) iPSC line and SOD1G128R (H) iPSC line.

Additional file 6: Supplementary Figure 3. pCyto-roGFP2-Orp1-donor (A) and pMitoroGFP2-Orp1-donor (B) plasmid maps. Schematic maps of the donor plasmids used for CRISPR/Cas 9 target insertion of the biosensors in the AAVS1 locus. Basic elements: homologous arms, puromycine resistance gene, promoters, Tet-On elements for doxycycline-controllable expression, and biosensors' functional elements are present. All maps were made with SnapGene ${ }^{\circledR}$. 
Additional file 7: Supplementary Figure 4 SOX2 and SSEA4 staining of the transgenic iPSC clones expressing Cyto-roGFP2-Orp1 (_Cyto) or Mito-roGFP2-Orp1 (_Mito) biosensors. Nuclei are visualized with DAPI, scale bar $100 \mu \mathrm{M}$.

Additional file 8: Supplementary Figure 5 Immunofluorescent staining of iPSC-derived motor neurons (differentiation day 28) for ChAT, MNX1, and ISL1. Nuclei are visualized with DAPI. Scale bar $200 \mu \mathrm{M}$.

\section{ACKNOWLEDGEMENTS}

Authors wish to thank the Microscopy Center of Biological Objects of the Siberian Branch of the Russian Academy of Sciences for granting access to microscopic equipment and SB RAS Genomics Core Facility for Sanger sequencing of the samples.

\section{FUNDING}

This work was supported by the State project of the Institute of Cytology and Genetics \# 02592021-0011.

\section{AUTHORS' CONTRIBUTIONS}

EIU and SPM designed the research. EIU performed the experiments and analyzed the data. SPM and SMZ obtained the funding. EIU drafted the manuscript and SPM and SMZ revised and approved it. All authors have read and agreed to the published version of the manuscript.

\section{AVAILABILITY OF DATA AND MATERIALS}

The datasets used and/or analyzed during the current study are available from the corresponding authors on reasonable request. 


\section{ETHICS APPROVAL AND CONSENT TO PARTICIPATE}

The work regarding genetic modification of the iPSC lines, generated from the patients' materials has been approved by the Research Ethics Committee of FSBI Federal Neurosurgical Center (Novosibirsk, Russia), protocol number 1 14/03/2017.

\section{CONSENT FOR PUBLICATION}

Not applicable.

\section{COMPETING INTERESTS}

The authors declare no competing interests.

\section{REFERENCES}

1. Dias V, Junn E, Mouradian MM. The role of oxidative stress in parkinson's disease. J Parkinsons Dis. 2013;3:461-91. https://doi.org/10.3233/JPD-130230.

2. Barber SC, Shaw PJ. Oxidative stress in ALS: Key role in motor neuron injury and therapeutic $\begin{array}{llll}\text { target. } & \text { Free } & \text { Radic } & \text { Biol }\end{array}$ Med. https://doi.org/10.1016/j.freeradbiomed.2009.11.018

3. Chinta SJ, Andersen JK. Redox imbalance in Parkinson's disease. Biochim Biophys Acta Gen Subj. 2008;1780:1362-7. https://doi.org/10.1016/j.bbagen.2008.02.005.

4. Esposito S, Masala A, Sanna S, Rassu M, Pimxayvong V, Iaccarino C, et al. Redox-sensitive GFP to monitor oxidative stress in neurodegenerative diseases. Rev Neurosci. 2017;28:133-44. https://doi.org/10.1515/revneuro-2016-0041.

5. Malkus KA, Tsika E, Ischiropoulos H. Oxidative modifications, mitochondrial dysfunction, and impaired protein degradation in Parkinson's disease: How neurons are lost in the Bermuda triangle. Mol Neurodegener. 2009;4:24. https://doi.org/10.1186/1750-1326-4-24. 
6. Machiela E, Jeloka R, Caron NS, Mehta S, Schmidt ME, Baddeley HJE, et al. The Interaction of Aging and Cellular Stress Contributes to Pathogenesis in Mouse and Human Huntington $\begin{array}{lllll}\text { Disease } & \text { Neurons. } & \text { Front } & \text { Aging } & \text { Neurosci. }\end{array}$ https://doi.org/10.3389/fnagi.2020.524369.

7. Vance C, Scotter EL, Nishimura AL, Troakes C, Mitchell JC, Kathe C, et al. ALS mutant FUS disrupts nuclear localization and sequesters wild-type FUS within cytoplasmic stress granules. Hum Mol Genet. 2013;22:2676-88. https://doi.org/10.1093/hmg/ddt117.

8. Ustyantseva EI, Medvedev SP, Zakian SM. Studying ALS: Current Approaches, Effect on Potential Treatment Strategy. Adv Exp Med Biol. 2020;1241:195-217. https://doi.org/10.1007/978-3-030-41283-8_11.

9. Rosen R. D, Siddique T, Patterson D, Figlewicz A. D, Sapp P, Hentati A. Mutations in Cu/Zn superoxide dismutase gene are associated with familial amyotrophic lateral sclerosis. Nature. 1993;362:59-62. https://doi.org/10.1038/362059a0.

10. Chen H, Kankel MW, Su SC, Han SWS, Ofengeim D. Exploring the genetics and non-cell autonomous mechanisms underlying ALS/FTLD. Cell Death Differ. 2018;25:646-60. https://doi.org/10.1038/s41418-018-0060-4.

11. Zhang K, Donnelly CJ, Haeusler AR, Grima JC, Machamer JB, Steinwald P, et al. The C9orf72 repeat expansion disrupts nucleocytoplasmic transport. Nature. 2015;525:56-61. https://doi.org/10.1038/nature14973.

12. Bilican B, Serio A, Barmada SJ, Nishimura AL, Sullivan GJ, Carrasco M, et al. Mutant induced pluripotent stem cell lines recapitulate aspects of TDP-43 proteinopathies and reveal cell-specific vulnerability. Proc Natl Acad Sci USA. 2012;109:5803-8. https://doi.org/10.1073/pnas.1202922109.

13. Gurney ME. The use of transgenic mouse models of amyotrophic lateral sclerosis in preclinical drug studies. J Neurol Sci. 1997;152 Suppl 1:67-73. https://doi.org/10.1016/s0022$510 x(97) 00247-5$. 
14. Bosco DA, Morfini G, Karabacak NM, Song Y, Gros-Louis F, Pasinelli P, et al. Wild-type and mutant SOD1 share an aberrant conformation and a common pathogenic pathway in ALS. Nat Neurosci. 2010;13:1396-403. https://doi.org/10.1038/nn.2660.

15. Fang C, Bourdette D, Banker G. Oxidative stress inhibits axonal transport: Implications for neurodegenerative diseases. Mol Neurodegener. 2012;7:29. https://doi.org/10.1186/1750-13267-29.

16. Rojas F, Gonzalez D, Cortes N, Ampuero E, Hern $\tilde{A}_{j}$ ndez DE, Fritz E, et al. Reactive oxygen species trigger motoneuron death in non-cell-autonomous models of ALS through activation of c-Abl signaling. Front Cell Neurosci. 2015;09:203. https://doi.org/10.3389/fncel.2015.00203.

17. Fischer LR, Li Y, Asress SA, Jones DP, Glass JD. Absence of SOD1 leads to oxidative stress in peripheral nerve and causes a progressive distal motor axonopathy. Exp Neurol. 2012;233:163-71. https://doi.org/10.1016/j.expneurol.2011.09.020.

18. Richardson K, Allen SP, Mortiboys H, Grierson AJ, Wharton SB, Ince PG, et al. The effect of SOD1 mutation on cellular bioenergetic profile and viability in response to oxidative stress and influence of mutation-type. PLoS One. 2013;8:e68256. https://doi.org/10.1371/journal.pone.0068256.

19. Jiang X, Wang L, Carroll SL, Chen J, Wang MC, Wang J. Challenges and opportunities for small-molecule fluorescent probes in redox biology applications. Antioxidants Redox Signal. 2018;29:518-40. https://doi.org/10.1089/ars.2017.7491.

20. Tarpey MM, Wink DA, Grisham MB. Methods for detection of reactive metabolites of oxygen and nitrogen: In vitro and in vivo considerations. Am J Physiol - Regul Integr Comp Physiol. 2004;286(3):R431-44. https://doi.org/10.1152/ajpregu.00361.

21. Wolf AM, Nishimaki K, Kamimura N, Ohta S. Real-time monitoring of oxidative stress in live mouse skin. J Invest Dermatol. 2014;134:1701-9. https://doi.org/10.1038/jid.2013.428.

22. Meyer AJ, Brach T, Marty L, Kreye S, Rouhier N, Jacquot JP, et al. Redox-sensitive GFP in Arabidopsis thaliana is a quantitative biosensor for the redox potential of the cellular glutathione 
redox buffer. Plant J. 2007;52:973-86. https://doi.org/10.1111/j.1365-313X.2007.03280.x.

23. Panieri E, Millia C, Santoro MM. Real-time quantification of subcellular $\mathrm{H} 2 \mathrm{O} 2$ and glutathione redox potential in living cardiovascular tissues. Free Radic Biol Med. 2017;109:189200. https://doi.org/10.1016/j.freeradbiomed.2017.02.022.

24. Fujikawa Y, Roma LP, Sobotta MC, Rose AJ, Diaz MB, Locatelli G, et al. Mouse redox histology using genetically encoded probes. Sci Signal. 2016;9 (419):rs1. https://doi.org/10.1126/scisignal.aad3895.

25. Du Y, Hu H, Pei X, Du K, Wei T. Genetically Encoded FapR-NLuc as a Biosensor to Determine Malonyl-CoA in Situ at Subcellular Scales. Bioconjug Chem. 2019;30:826-32. https://doi.org/10.1021/acs.bioconjchem.8b00920.

26. Duprez J, Roma LP, Close AF, Jonas JC. Protective Antioxidant and Antiapoptotic Effects of $\mathrm{ZnCl} 2$ in Rat Pancreatic Islets Cultured in Low and High Glucose Concentrations. PLoS One. 2012;7 (10):e46831. https://doi.org/10.1371/journal.pone.0046831.

27. Desireddi JR, Farrow KN, Marks JD, Waypa GB, Schumacker PT. Hypoxia increases ros signaling and cytosolic $\mathrm{Ca} 2+$ in pulmonary artery smooth muscle cells of mouse lungs slices. Antioxidants Redox Signal. 2010;12:595-602. https://doi.org/10.1089/ars.2009.2862.

28. Van Hameren G, Campbell G, Deck M, Berthelot J, Gautier B, Quintana P, et al. In vivo real-time dynamics of ATP and ROS production in axonal mitochondria show decoupling in mouse models of peripheral neuropathies. Acta Neuropathol Commun. Acta Neuropathologica Communications; 2019;7:86. https://doi.org/10.1186/s40478-019-0740-4.

29. Ustyantseva EI, Medvedev SP, Vetchinova AS, Minina JM, Illarioshkin SN, Zakian SM. A Platform for Studying Neurodegeneration Mechanisms Using Genetically Encoded Biosensors. Biochemistry. 2019;84:299-309. https://doi.org/10.1134/S000629791903012X.

30. Malakhova AA, Grigor'eva E V., Pavlova S V., Malankhanova TB, Valetdinova KR, Vyatkin Y V., et al. Generation of induced pluripotent stem cell lines ICGi021-A and ICGi022-A from peripheral blood mononuclear cells of two healthy individuals from Siberian population. 
Stem Cell Res. 2020;48:101952. https://doi.org/10.1016/j.scr.2020.101952.

31. Hsu PD, Scott DA, Weinstein JA, Ran FA, Konermann S, Agarwala V, et al. DNA targeting specificity of RNA-guided Cas9 nucleases. Nat Biotechnol. 2013;31:827-32. https://doi.org/10.1038/nbt.2647.

30. Jinek M, Chylinski K, Fonfara I, Hauer M, Doudna JA, Charpentier E. A Programmable Dual-RNA-Guided DNA Endonuclease in Adaptive Bacterial Immunity. 2012;337:816-21. https://doi.org/10.1126/science.1225829.

32. Jinek M, Chylinski K, Fonfara I, Hauer M, Doudna JA, Charpentier E. A Programmable Dual-RNA-Guided DNA Endonuclease in Adaptive Bacterial Immunity. 2012;337:816-21. https://doi.org/10.1126/science.1225829.

33. Medrano RFV, De Oliveira CA. Guidelines for the tetra-primer ARMS-PCR technique development. Mol Biotechnol. 2014;56:599-608. https://doi.org/10.1007/s12033-014-9734-4.

34. Ustyantseva EI, Medvedev SP, Vetchinova AS, Illarioshkin SN, Leonov S V., Zakian SM. Generation of an induced pluripotent stem cell line, ICGi014-A, by reprogramming peripheral blood mononuclear cells from a patient with homozygous D90A mutation in SOD1 causing Amyotrophic lateral sclerosis. Stem Cell Res. 2020;42:101675. https://doi.org/10.1016/j.scr.2019.101675.

35. Du Z-W, Chen H, Liu H, Lu J, Qian K, Huang C-L, et al. Generation and expansion of highly pure motor neuron progenitors from human pluripotent stem cells. Nat Commun2015;6:6626. https://doi.org/10.1038/ncomms7626.

36. Morgan B, Sobotta MC, Dick TP. Measuring EGSHand H2O2with roGFP2-based redox probes. Free Radic Biol Med. 2011;51:1943-51. https://doi.org/ 10.1016/j.freeradbiomed.2011.08.035.

37. Picher-Martel V, Valdmanis PN, Gould P V, Julien J-P, Dupré N. From animal models to human disease: a genetic approach for personalized medicine in ALS. Acta Neuropathol Commun. 2016;4:70. https://doi.org/10.1186/s40478-016-0340-5. 
38. Alemasov NA, Ivanisenko N V., Ramachandran S, Ivanisenko VA. Molecular mechanisms underlying the impact of mutations in SOD1 on its conformational properties associated with amyotrophic lateral sclerosis as revealed with molecular modelling. BMC Struct Biol. 2018;18:1-14. https://doi.org/10.1186/s12900-018-0080-9.

39. Pereira GRC, De Azevedo Abrahim Vieira B, De Mesquita JF. Comprehensive in silico analysis and molecular dynamics of the superoxide dismutase 1 (SOD1) variants related to amyotrophic lateral sclerosis. PLoS One. 2021;16(2):e0247841. https://doi.org/10.1371/journal.pone.0247841.

40. Luigetti M, Conte A, Madia F, Marangi G, Zollino M, Mancuso I, et al. Heterozygous SOD1 D90A mutation presenting as slowly progressive predominant upper motor neuron amyotrophic lateral sclerosis. Neurol Sci. 2009;30:517-20. https://doi.org/10.1007/s10072-009-0125-8.

41. Holmøy T, Wilson JA, Von Der Lippe C, Andersen PM, Berg-Hansen P. G127R: A novel SOD1 mutation associated with rapidly evolving ALS and severe pain syndrome. Amyotroph Lateral Scler. 2010;11:478-80. https://doi.org/10.3109/17482960903580315.

42. Das AT, Tenenbaum L, Berkhout B. Tet-On Systems For Doxycycline-inducible Gene $\begin{array}{llll}\text { Expression. } & \text { Curr } & \text { The } & \text { Ther. }\end{array}$ https://doi.org/10.2174/1566523216666160524144041

43. DeKelver RC, Choi VM, Moehle EA, Paschon DE, Hockemeyer D, Meijsing SH, et al. Functional genomics, proteomics, and regulatory DNA analysis in isogenic settings using zinc finger nuclease-driven transgenesis into a safe harbor locus in the human genome. Genome Res. 2010;20:1133-42. https://doi.org/10.1101/gr.106773.110.

44. Kotin RM, Linden RM, Berns KI. Characterization of a preferred site on human chromosome 19q for integration of adeno-associated virus DNA by non-homologous recombination. EMBO J. $1992 ; 11: 5071-8$.

45. Li E. Chromatin modification and epigenetic reprogramming in mammalian development. Nat Rev Genet. 2002;3:662-73. https://doi.org/10.1038/nrg887. 
46. Schwarzländer M, Dick TP, Meyer AJ, Morgan B. Dissecting Redox Biology using Fluorescent Protein Sensors. Antioxid Redox Signal. 2016;24:680-712. https://doi.org/10.1089/ars.2015.6266.

47. Albrecht SC, Barata AG, Großhans J, Teleman AA, Dick TP. In vivo mapping of hydrogen peroxide and oxidized glutathione reveals chemical and regional specificity of redox homeostasis. Cell Metab. 2011;14:819-29. https://doi.org/10.1016/j.cmet.2011.10.010.

48. Zhao S, Li L, Wang S, Yu C, Xiao B, Lin L, et al. H2O2 treatment or serum deprivation induces autophagy and apoptosis in naked mole-rat skin fibroblasts by inhibiting the PI3K/Akt signaling pathway. Oncotarget. 2016;7:84839-50. https://doi.org/10.18632/oncotarget.13321.

49. Le Gall L, Anakor E, Connolly O, Vijayakumar UG, Duddy WJ, Duguez S. Molecular and cellular mechanisms affected in ALS. J Pers Med. 2020;10:101. https://doi.org/10.3390/jpm10030101.

50. Nicholls DG, Budd SL. Mitochondria and neuronal glutamate excitotoxicity. Biochim Biophys Acta - Bioenerg. 1998;1366:97-112. https://doi.org/10.1016/S0005-2728(98)00123-6.

51. Carriedo SG, Sensi SL, Yin HZ, Weiss JH. AMPA exposures induce mitochondrial Ca2+ overload and ROS generation in spinal motor neurons in vitro. J Neurosci. 2000;20:240-50. https://doi.org/10.1523/jneurosci.20-01-00240.2000.

52. Sun YH, Kao HKJ, Chang CW, Merleev A, Overton JL, Pretto D, et al. Human induced pluripotent stem cell line with genetically encoded fluorescent voltage indicator generated via CRISPR for action potential assessment post-cardiogenesis. Stem Cells. 2020;38:90-101. https://doi.org/10.1002/stem.3085.

53. Guha S, Fischer S, Johnson GVW, Nehrke K. Tauopathy-associated tau modifications selectively impact neurodegeneration and mitophagy in a novel C. elegans single-copy transgenic model. Mol Neurodegener. Molecular Neurodegeneration; 2020;15:65. https://doi.org/10.1186/s13024-020-00410-7.

54. Sieger D, Moritz C, Ziegenhals T, Prykhozhij S, Peri F. Long-Range Ca2+ Waves Transmit 
Brain-Damage Signals to Microglia. Dev Cell. 2012;22:1138-48. https://doi.org/10.1016/j.devcel.2012.04.012.

55. Gao L, Arias-Mayenco I, Ortega-Sáenz P, López-Barneo J. Using redox-sensitive fluorescent probes to record real-time reactive oxygen species production in cells from mouse carotid body slices. STAR Protoc. 2021;2: 100535. https://doi.org/10.1016/j.xpro.2021.100535.

56. Greenwald EC, Mehta S, Zhang J. Genetically Encoded Fluorescent Biosensors Illuminate the Spatiotemporal Regulation of Signaling Networks. Chem Rev. 2018;118:11707-11794. https://doi.org/10.1021/acs.chemrev.8b00333.

57. Dabirian Y, Li X, Chen Y, David F, Nielsen J, Siewers V. Expanding the Dynamic Range of a Transcription Factor-Based Biosensor in Saccharomyces cerevisiae. ACS Synth Biol. American Chemical Society; 2019;8:1968-75. doi: 10.1021/acssynbio.9b00144.

58. Zhu P, Aller MI, Baron U, Cambridge S, Bausen M, Herb J, et al. Silencing and Un-silencing of Tetracycline-Controlled Genes in Neurons. PLoS One. 2007;2:e533. https://doi.org/10.1371/journal.pone.0000533.

59. Ordovás L, Boon R, Pistoni M, Chen Y, Wolfs E, Guo W, et al. Efficient recombinasemediated cassette exchange in hPSCs to study the hepatocyte lineage reveals AAVS1 locusmediated transgene inhibition. Stem Cell Reports. 2015;5:918-31. https://doi.org/10.1016/j.stemcr.2015.09.004.

60. Klatt D, Cheng E, Hoffmann Di, Santilli G, Thrasher AJ, Brendel C, et al. Differential Transgene Silencing of Myeloid-Specific Promoters in the AAVS1 Safe Harbor Locus of Induced Pluripotent Stem Cell-Derived Myeloid Cells. Hum Gene Ther. 2020;31:199-210. https://doi.org/10.1089/hum.2019.194.

61. Li L, Chen Y, Gibson SB. Starvation-induced autophagy is regulated by mitochondrial reactive oxygen species leading to AMPK activation. Cell Signal. 2013;25:50-65. https://doi.org/10.1016/j.cellsig.2012.09.020

62. Wengrod J, Wang D, Weiss S, Zhong H, Osman I, Gardner LB. Phosphorylation of eIF2 $\alpha$ 
triggered by mTORC1 inhibition and PP6C activation is required for autophagy and is aberrant in PP6C-mutated melanoma. Sci $\quad$ Signal. 2015;8(367):ra27. https://doi.org/10.1126/scisignal.aaa0899.

63. Wengrod JC, Gardner LB. Cellular adaptation to nutrient deprivation: crosstalk between the mTORC1 and eIF2 $\alpha$ signaling pathways and implications for autophagy. Cell Cycle. 2015;14:2571-7. https://doi.org/10.1080/15384101.2015.1056947.

64. Marchetto MCN, Muotri AR, Mu Y, Smith AM, Cezar GG, Gage FH. Non-CellAutonomous Effect of Human SOD1G37R Astrocytes on Motor Neurons Derived from Human Embryonic Stem Cells. Cell Stem Cell. 2008;3:649-57. https://doi.org/10.1016/j.stem.2008.10.001

65. Pak V V., Ezeriņa D, Lyublinskaya OG, Pedre B, Tyurin-Kuzmin PA, Mishina NM, et al. Ultrasensitive Genetically Encoded Indicator for Hydrogen Peroxide Identifies Roles for the Oxidant in Cell Migration and Mitochondrial Function. Cell Metab. 2020;31:642-653. https://doi.org/10.1016/j.cmet.2020.02.003.

66. Rebs S, Sedaghat-Hamedani F, Kayvanpour E, Meder B, Streckfuss-Bömeke K. Generation of pluripotent stem cell lines and CRISPR/Cas9 modified isogenic controls from a patient with dilated cardiomyopathy harboring a RBM20 p.R634W mutation. Stem Cell Res. Elsevier; 2020;47:101901. https://doi.org/10.1016/j.scr.2020.101901.

67. Bursch F, Kalmbach N, Naujock M, Staege S, Eggenschwiler R, Abo-Rady M, et al. Altered calcium dynamics and glutamate receptor properties in iPSC-derived motor neurons from ALS patients with C9orf72, FUS, SOD1 or TDP43 mutations. Hum Mol Genet. 2019;28:2835-50. https://doi.org/10.1093/hmg/ddz107.

68. Van Damme P, Robberecht W. Clinical implications of recent breakthroughs in amyotrophic $\begin{array}{llll}\text { lateral } & \text { sclerosis. } & \text { Curr } & \text { Opin }\end{array}$ https://doi.org/10.1097/WCO.0b013e328364c063.

69. Tafuri F, Ronchi D, Magri F, Comi GP, Corti S. SOD1 misplacing and mitochondrial 
dysfunction in amyotrophic lateral sclerosis pathogenesis. Front Cell Neurosci. 2015;9:336. https://doi.org/10.3389/fncel.2015.00336

70. Millecamps S, Julien JP. Axonal transport deficits and neurodegenerative diseases. Nat Rev Neurosci. Nature Publishing Group; 2013;14:161-76. https://doi.org/10.1038/nrn3380. 
Figure 1

A

I. Generation of SOD1-mutant iPSCs

II. Generation of biosensors' expressing motor neurons
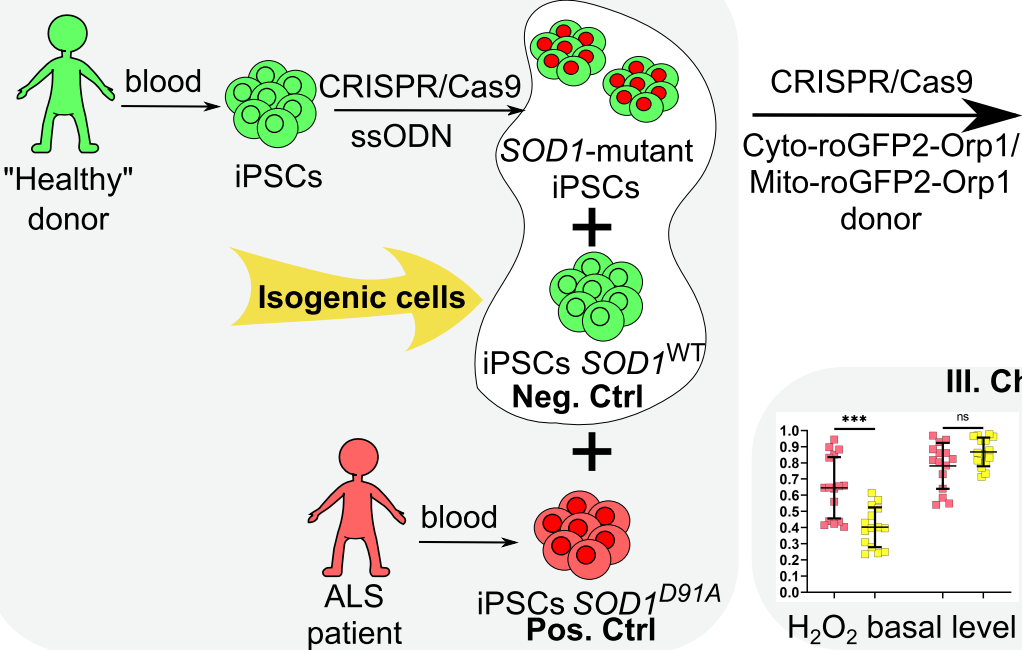

Mito-roGFP2-Orp1 donor

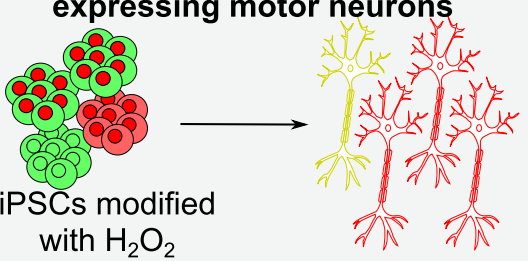

with $\mathrm{H}_{2} \mathrm{O}_{2}$

biosensors

Motor neurons

III. Characterisation and analysis
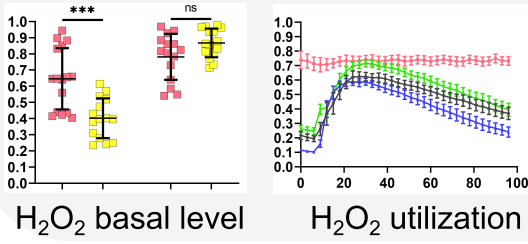

$\mathrm{H}_{2} \mathrm{O}_{2}$ utilization

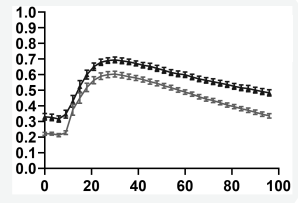

Stress-tests
B 5' CTTGGGCAATGTGACTGCTGACAAAGATGGTGTGGCCGAT 3' 3' GAACCCGTTACACTGACGACTGTTTCTACCACACCGGCTA 5'

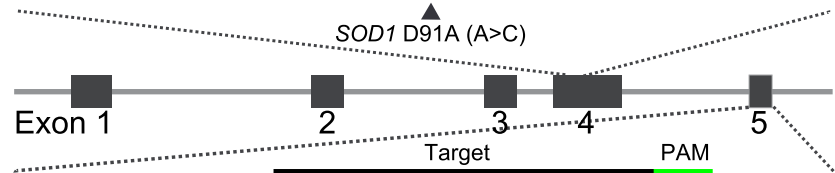

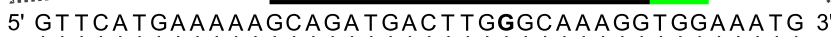

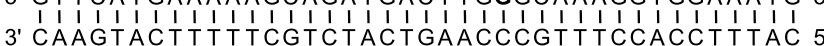
3. CAAGTACTTTTTCGTCTACTGAACCCGTTTCCACCTTTAC 5

SOD1 G128R (G>C)

SOD1-D91A
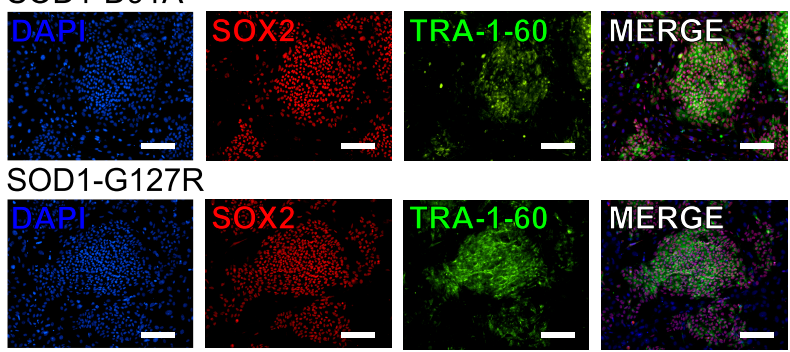

C

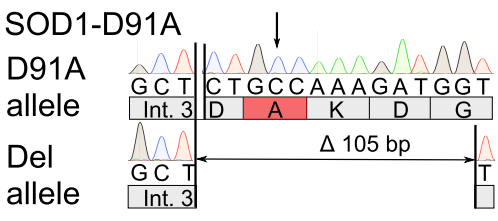

SOD1-G128R

G128R

allele

GACTTGCGCAAAGGTGGA

Stop

\begin{tabular}{|l|l|l|l|l|l|}
\hline$D$ & $L$ & $R$ & $K$ & $G$ & $G$ \\
\hline
\end{tabular}

allele

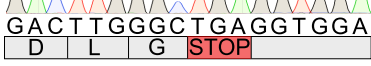

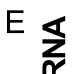

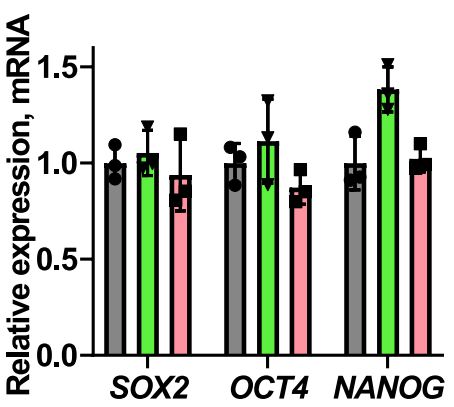

Alternative variants:

SOD1 D91AWT

SOD $1^{D 91 A / \Delta 5 b . p .}$

SOD $1^{D 91 A / i n s 1 b . p .}$

SOD1 $1^{\text {G128R/A5b.p. }}$

SOD $1^{G 128 R / \Delta 9 b . p .}$

SOD $1^{\text {G128R/ins. 1b.p. }}$

K7-4Lf

$\square$ SOD1-D91A

$\square$ SOD1-G128R 
Figure 2

A

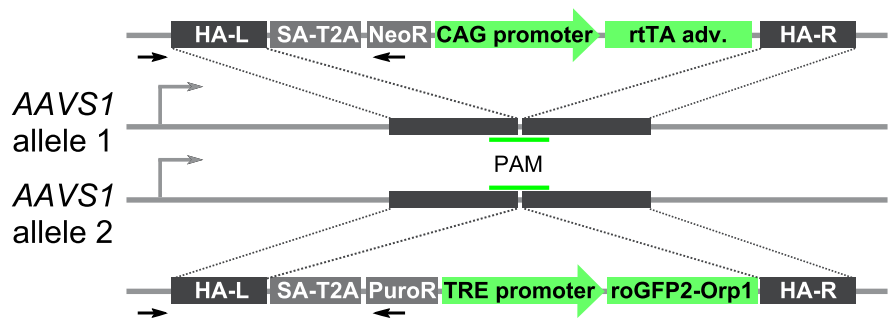

C

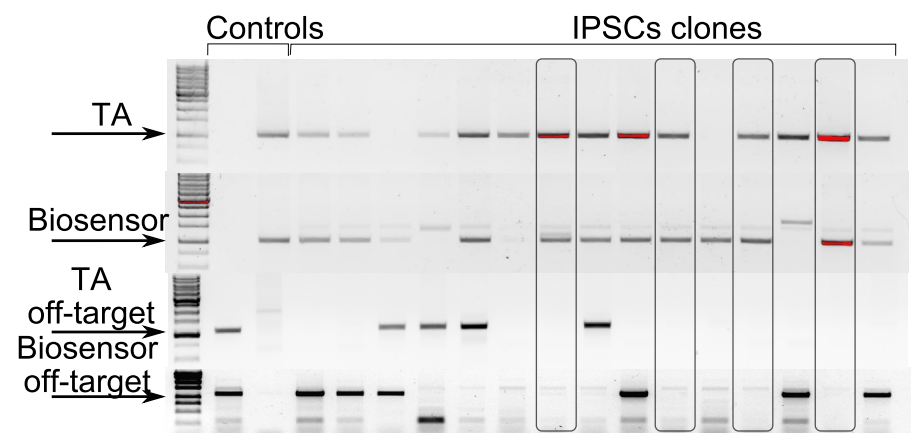

B
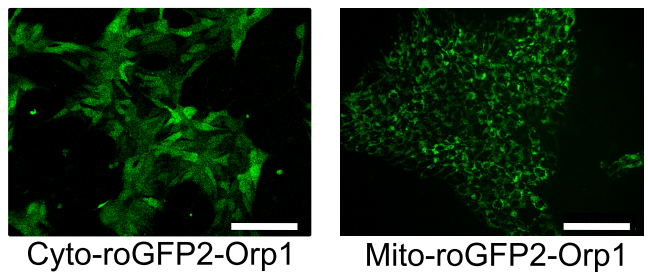

D

\begin{tabular}{|l|c|c|}
\hline \multicolumn{1}{|c|}{ iPSC line } & $\begin{array}{l}\text { Cyto-roGFP2- } \\
\text { Orp1 }\end{array}$ & $\begin{array}{l}\text { Mito-roGFP2- } \\
\text { Orp1 }\end{array}$ \\
\hline K7-4Lf & 5 clones & 3 clones \\
\hline SOD1-D91A & 14 clones & 11 clones \\
\hline SOD1-G128R & 12 clones & 12 clones \\
\hline iALS & 17 clones & 11 clones \\
\hline
\end{tabular}


Figure 4

A

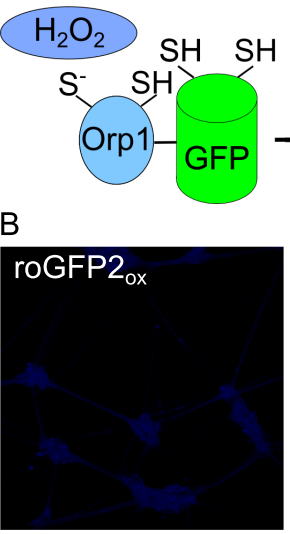

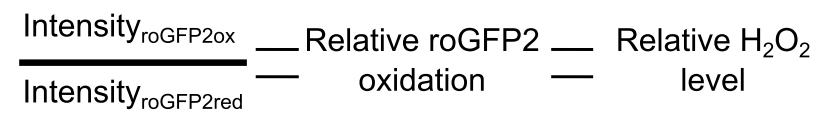

D
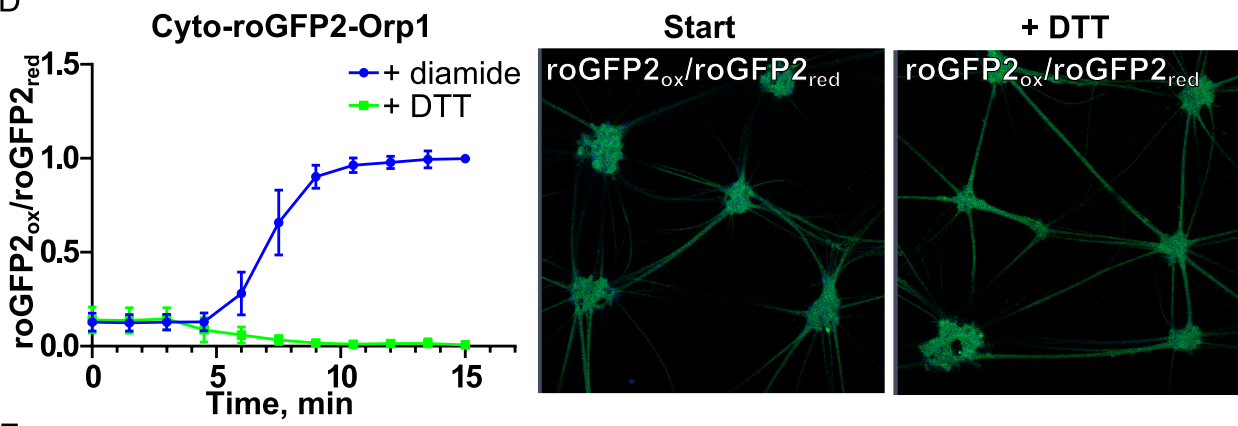

E
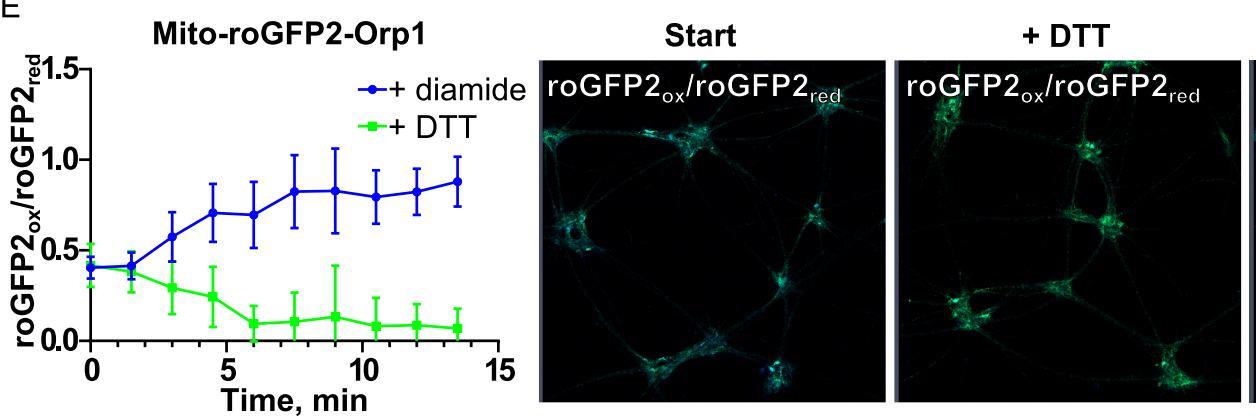

C

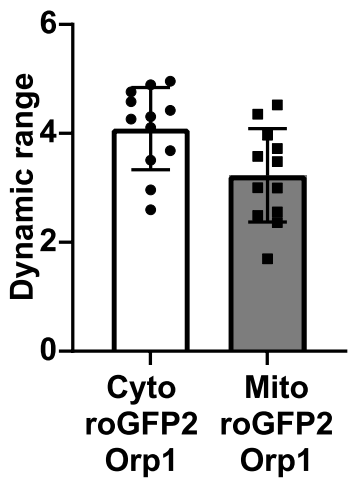

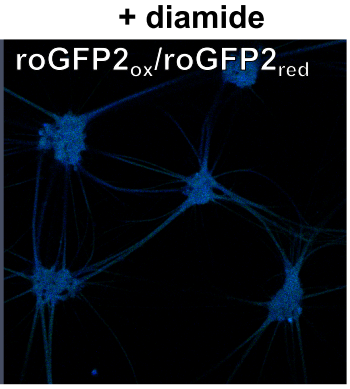

+diamide

roGFP $2_{\text {ox }} /$ roGFP $2_{\text {red }}$

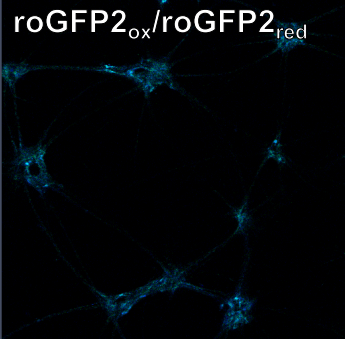


Figure 5

A

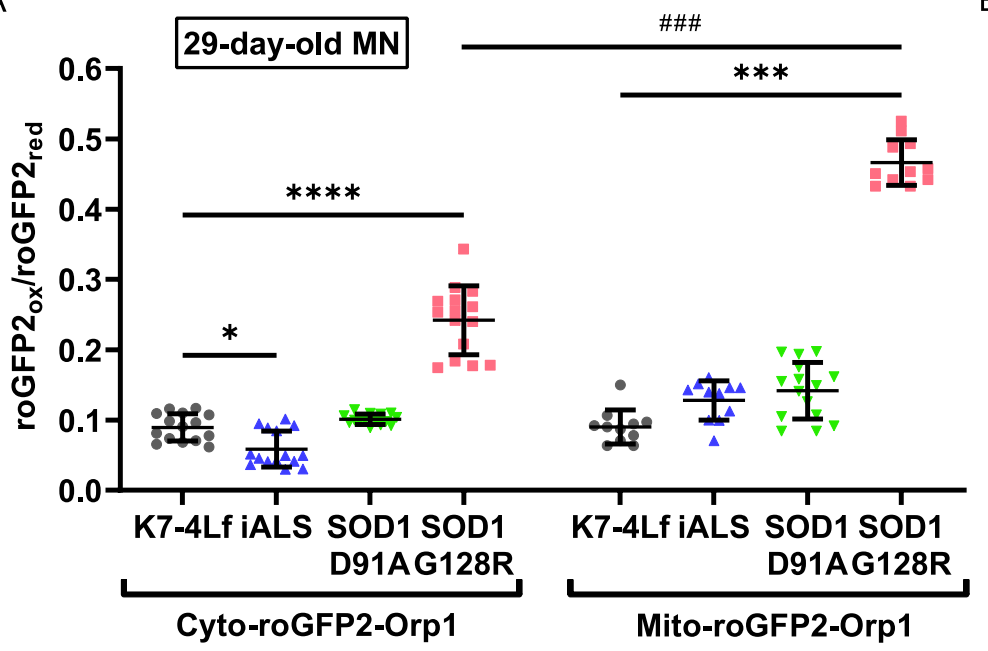

B

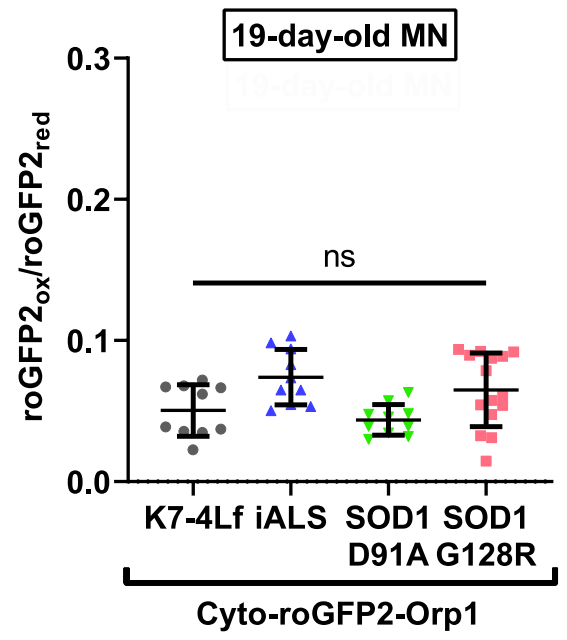

C

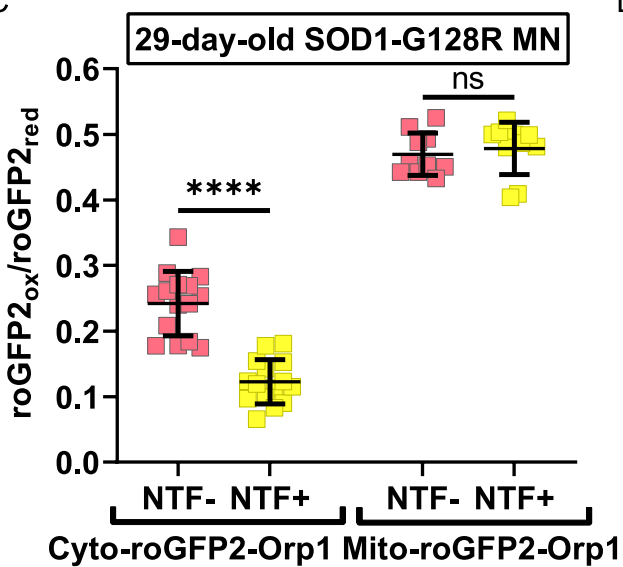

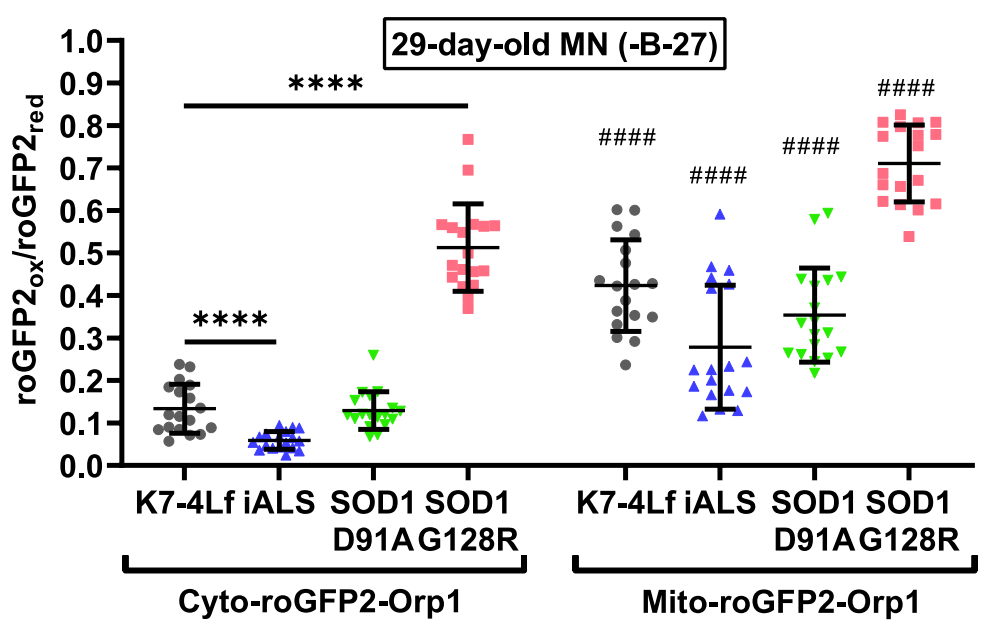


Figure 6

A

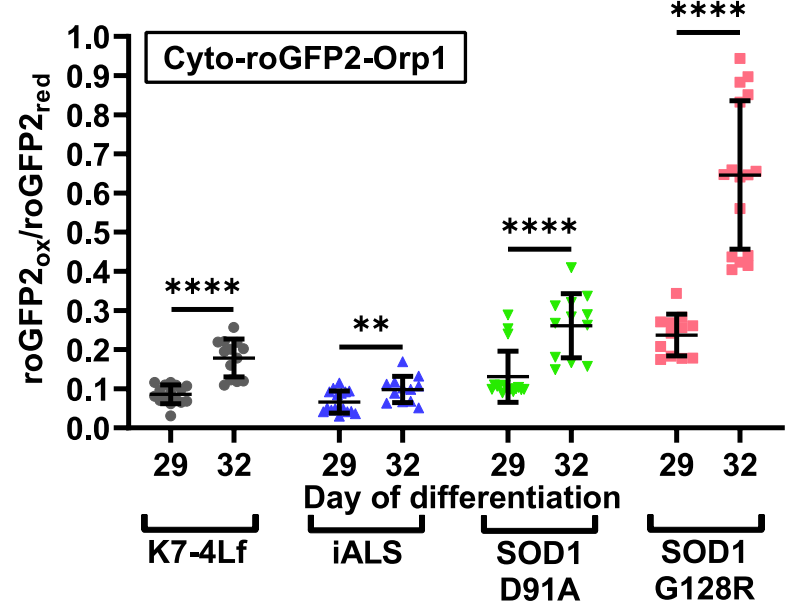

C

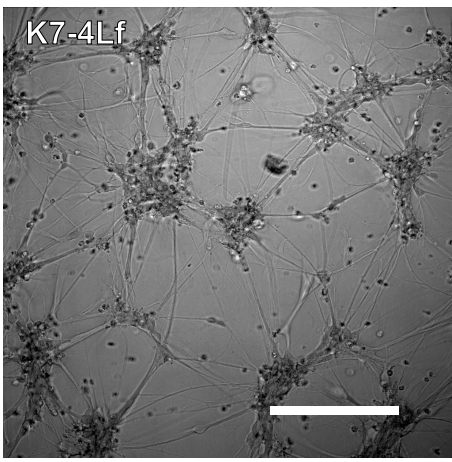

B
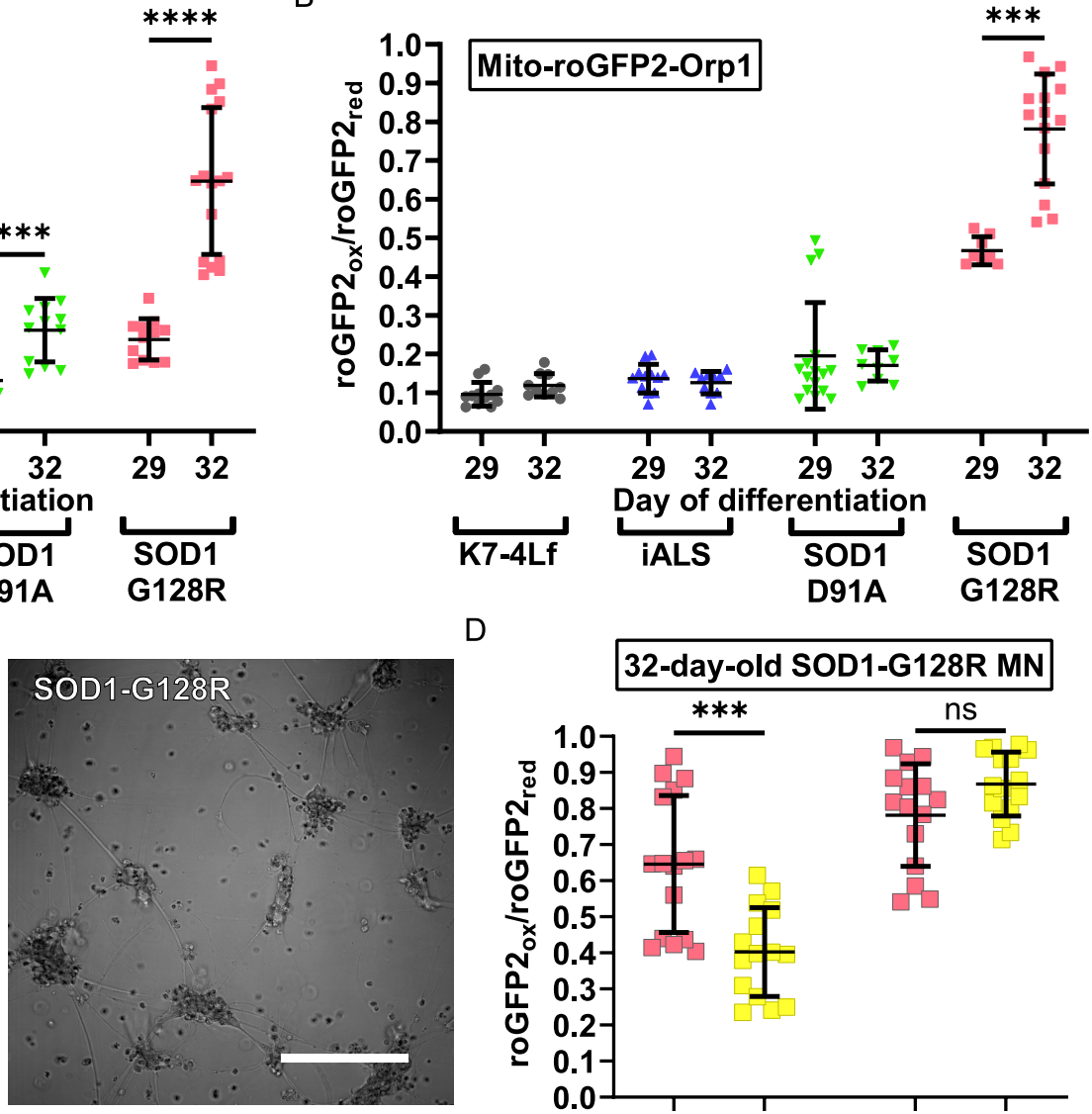

D

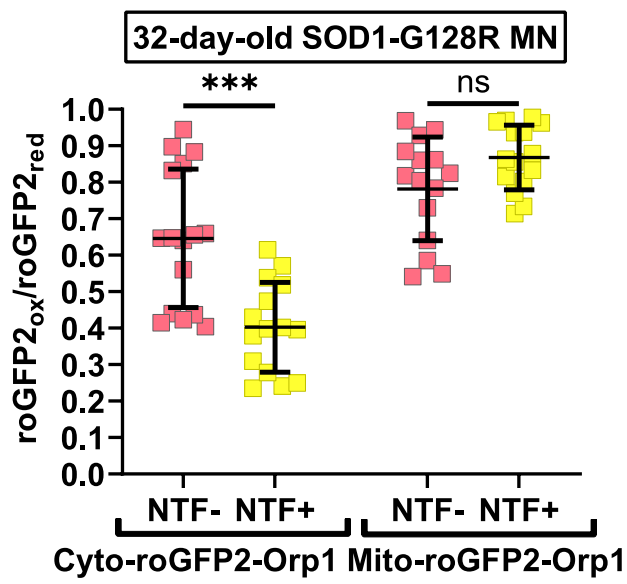


Figure 8

A

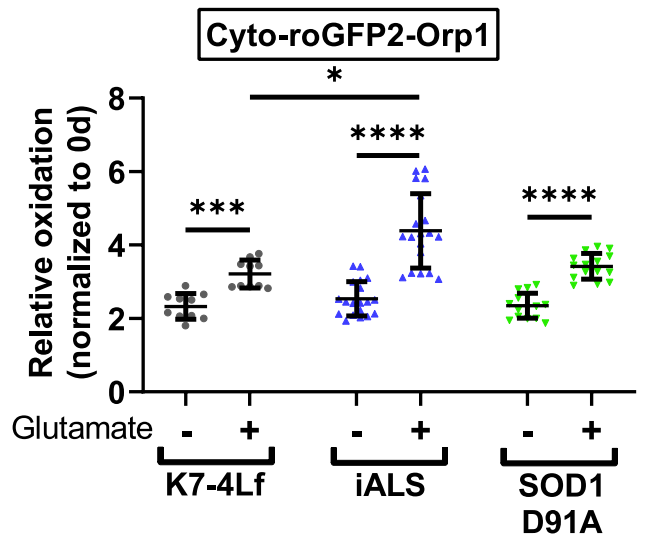

C

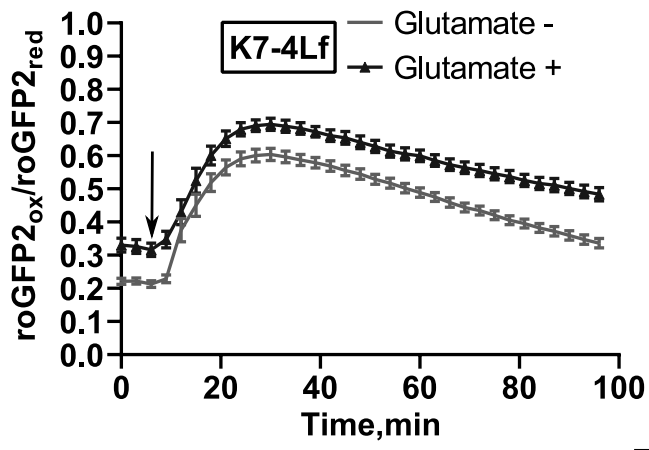

$E$

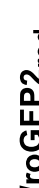

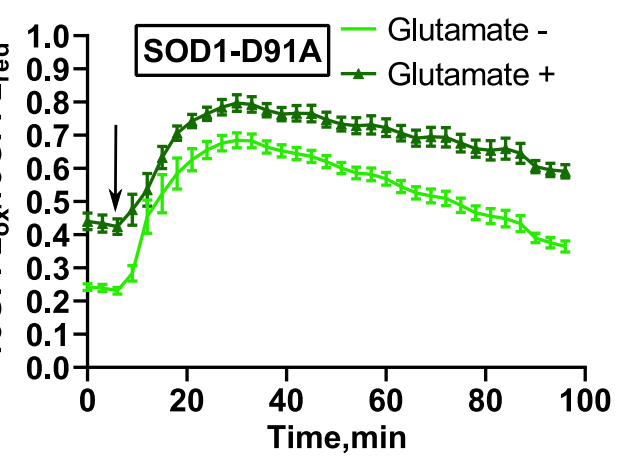

F
B
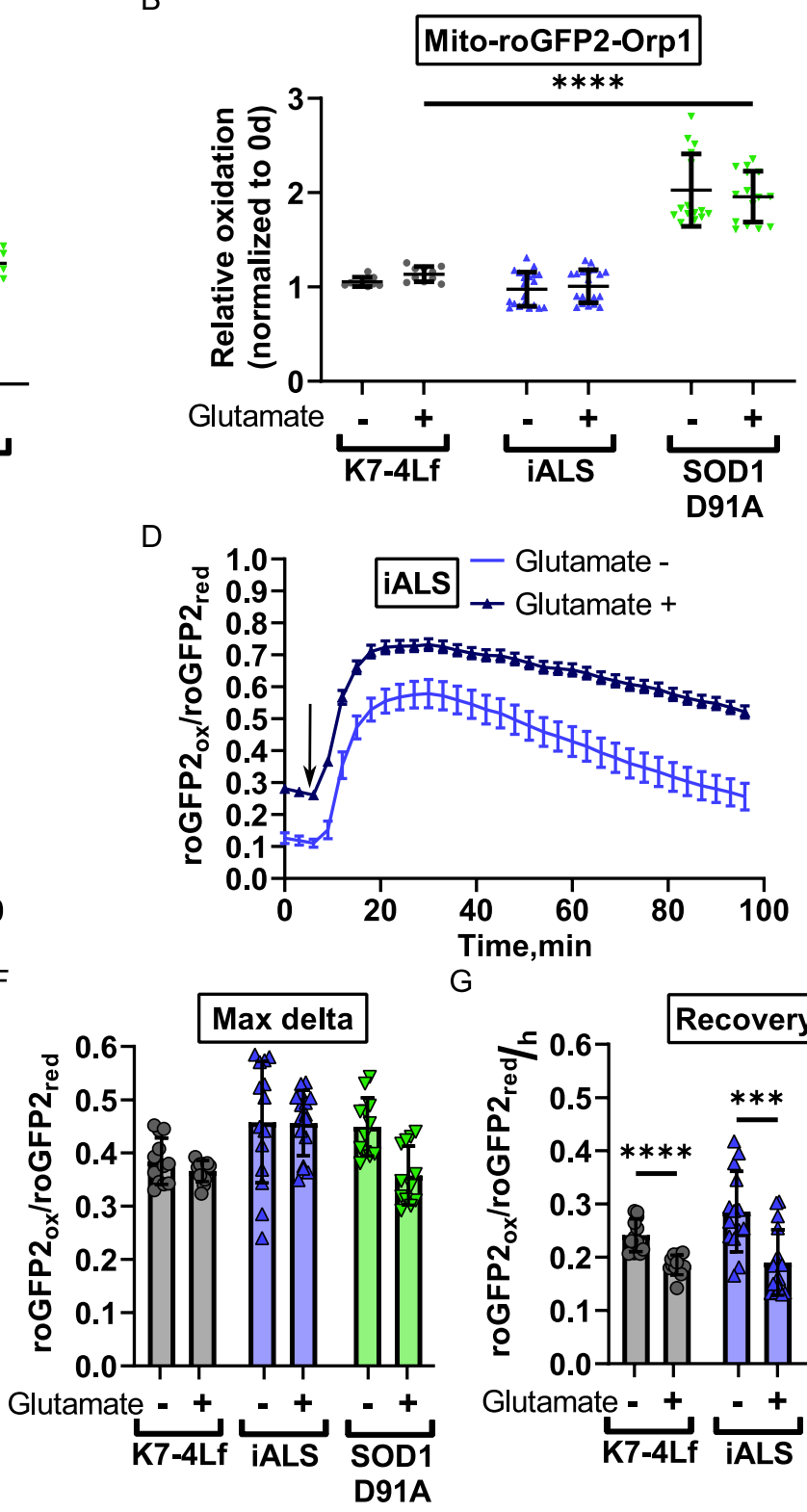

G

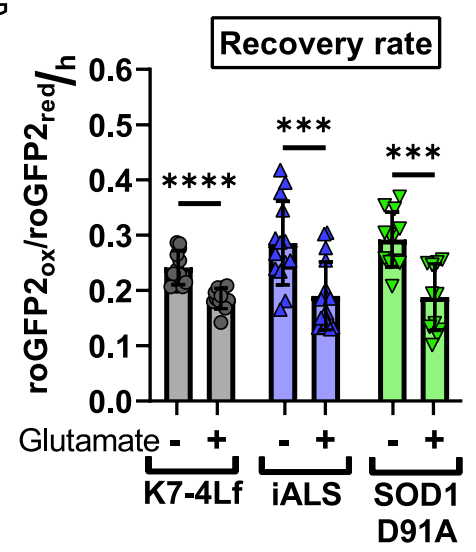

